\title{
Early Postpartum Awakenings, Neurocognitive Performance, and Feeding Methods
}

\author{
Michael A. Winser \\ West Virginia University
}

Follow this and additional works at: https://researchrepository.wvu.edu/etd

\section{Recommended Citation}

Winser, Michael A., "Early Postpartum Awakenings, Neurocognitive Performance, and Feeding Methods" (2012). Graduate Theses, Dissertations, and Problem Reports. 4939.

https://researchrepository.wvu.edu/etd/4939

This Thesis is protected by copyright and/or related rights. It has been brought to you by the The Research Repository @ WVU with permission from the rights-holder(s). You are free to use this Thesis in any way that is permitted by the copyright and related rights legislation that applies to your use. For other uses you must obtain permission from the rights-holder(s) directly, unless additional rights are indicated by a Creative Commons license in the record and/ or on the work itself. This Thesis has been accepted for inclusion in WVU Graduate Theses, Dissertations, and Problem Reports collection by an authorized administrator of The Research Repository @ WVU. For more information, please contact researchrepository@mail.wvu.edu. 
Early Postpartum Awakenings, Neurocognitive Performance, and Feeding Methods

Michael A. Winser

\author{
Thesis submitted to the \\ Eberly College of Arts and Sciences \\ at West Virginia University \\ in partial fulfillment of the requirements \\ for the degree of
}

\author{
Master of Science \\ in \\ Psychology
}
Hawley Montgomery-Downs, Ph.D., Chair Christina Duncan, Ph.D. Miranda Reed, Ph.D.
Department of Psychology

Morgantown, WestVirginia

2011

Keywords: Sleep, Breastfeeding, Prolactin, Psychomotor Vigilance Test, Reaction Time Copyright 2011 Michael A. Winser 


\begin{abstract}
Early Postpartum Awakenings, Neurocognitive Performance, and Feeding Methods
\end{abstract}

\title{
Michael A. Winser
}

This study investigated memory for brief nocturnal awakenings, characteristics of those awakenings in women during the early and late postpartum period, and how these awakenings were related to neurocognitive performance in exclusively breastfeeding and exclusively formula-feeding mothers.

The median duration of a nocturnal awakening necessary for morning recall was four minutes and 30 seconds, which closely resembles the current five-minute rule of thumb. There was normal variability in thresholds, indicating that other factors may influence memory for awakenings. During the postpartum period, women generally under-reported the number of awakenings experienced each night, but did increase in accuracy by week 12. Average number and duration of awakenings were not related to concurrent neurocognitive performance. There were no differences in number of lapses on the psychomotor vigilance test based on feeding method. However, exclusively formula-feeding mothers did have increased median reaction time overall compared to exclusively breastfeeding mothers, despite no significant differences in average number or duration of awakenings.

Findings from this work increase our basic understanding of memory formation during nocturnal awakenings, as well as how postpartum sleep disturbance changes over time and the associations between infant feeding method and maternal sleep disturbance. 


\section{TABLE OF CONTENTS}

TITLE PAGE .

ABSTRACT .

LIST OF TABLES

LIST OF FIGURES

LIST OF APPENDICES.

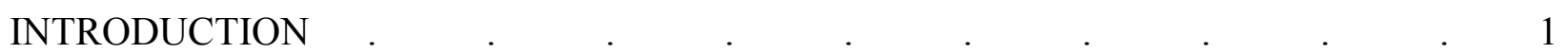

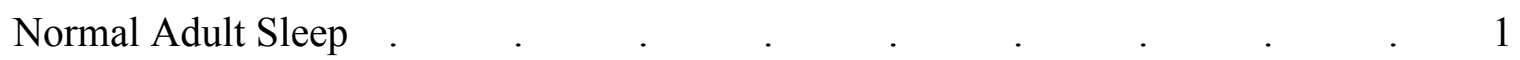

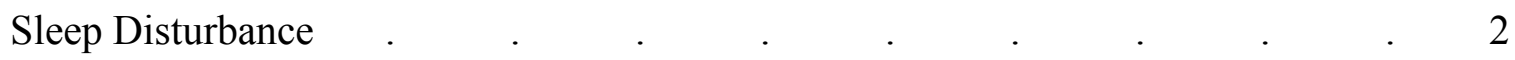

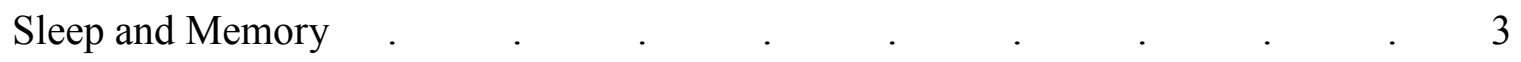

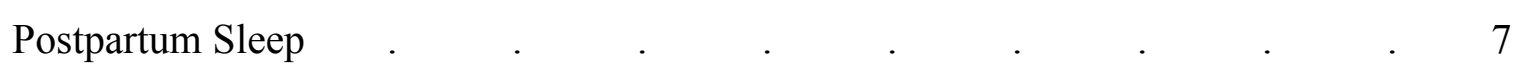

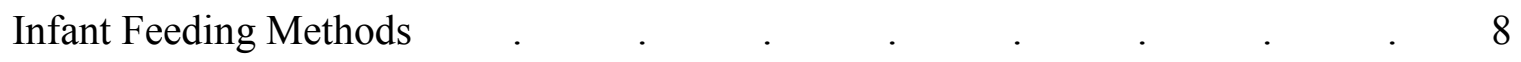

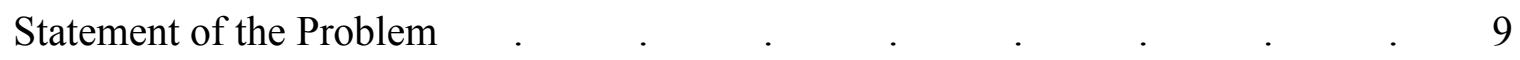

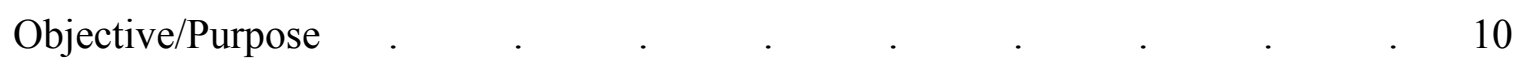

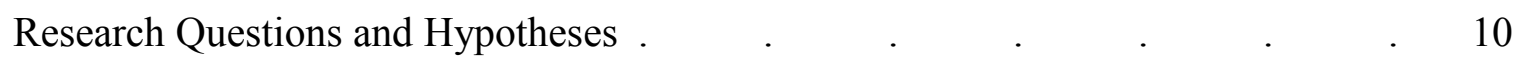

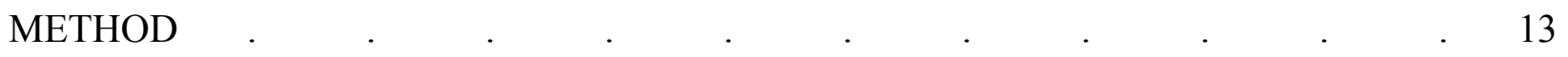

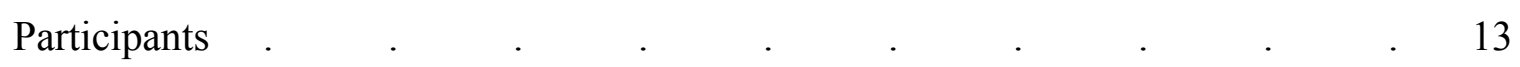

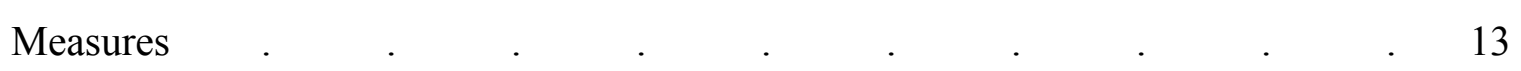

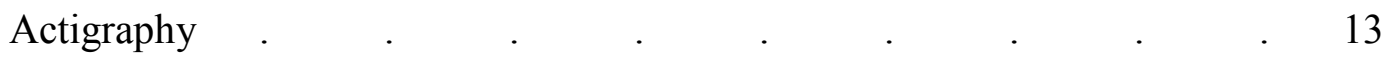

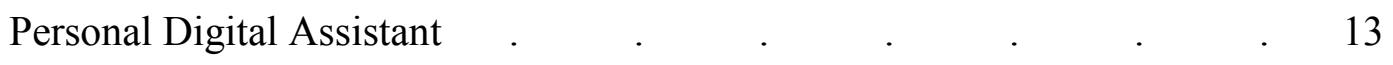

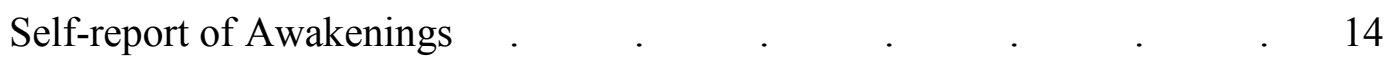

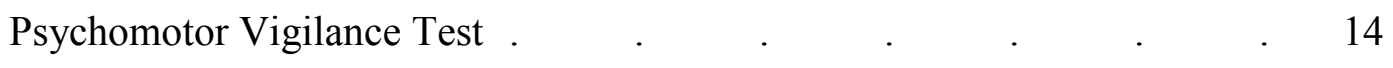

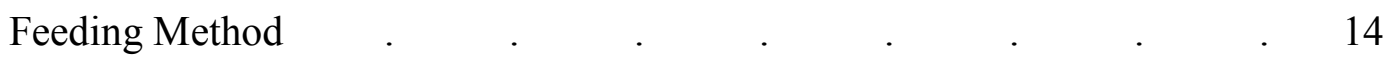

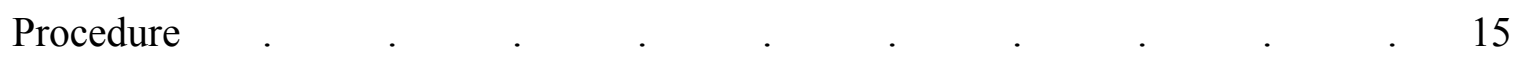

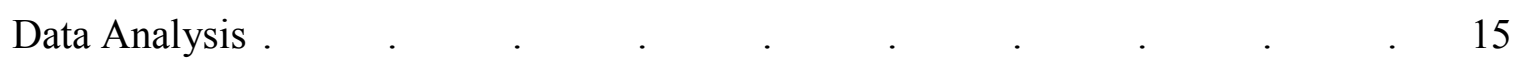




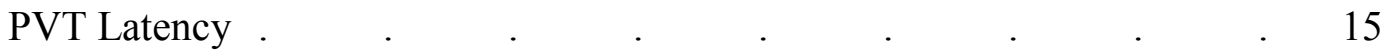

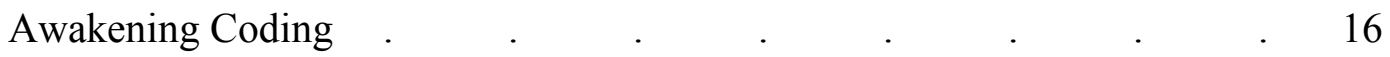

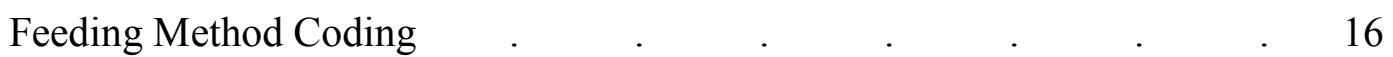

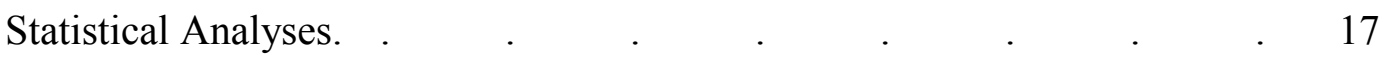

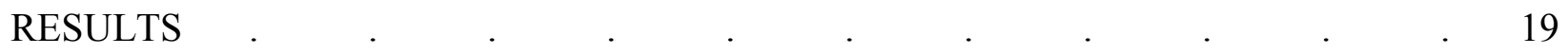

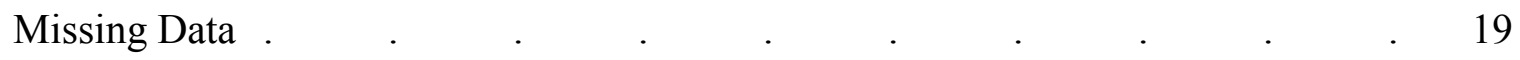

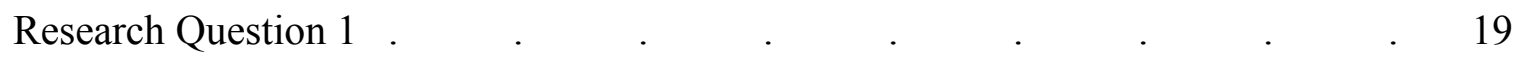

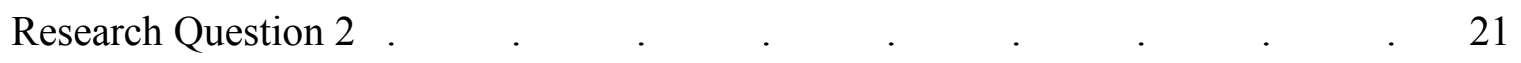

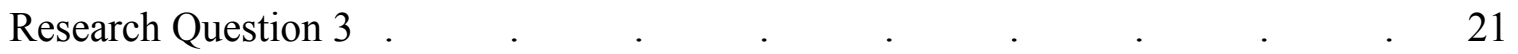

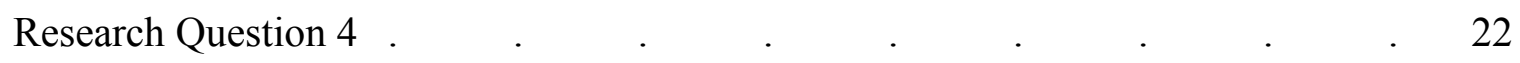

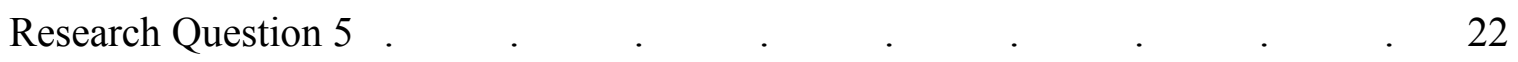

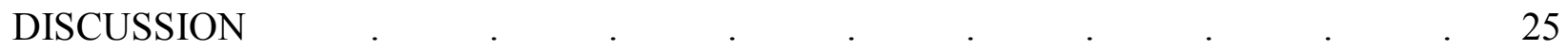

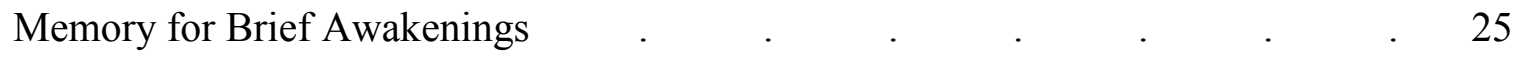

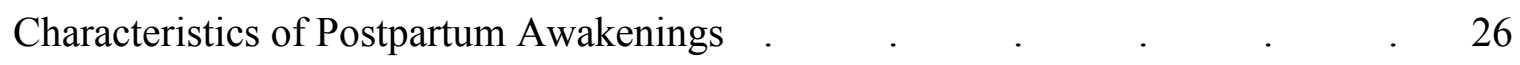

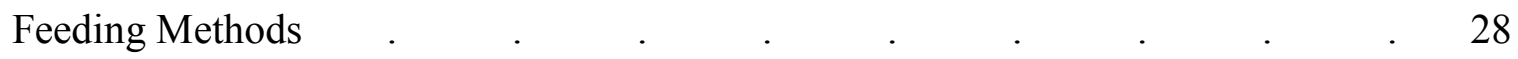

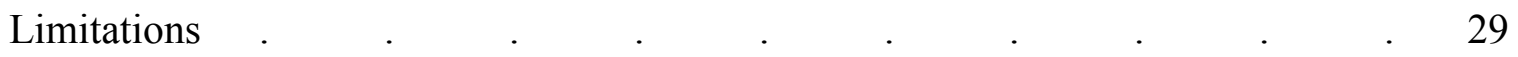

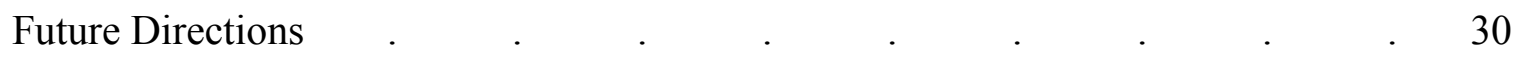

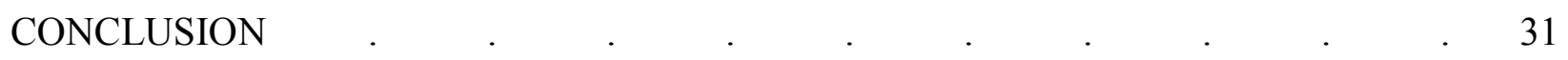

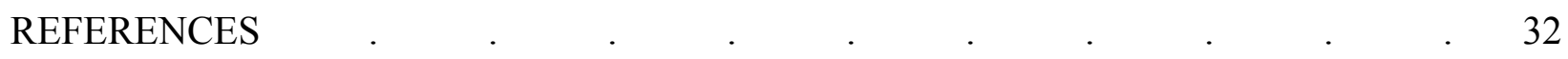




\section{LIST OF TABLES}

1. Summary of Studies Investigating Memory

during the Sleep/Wake Transition

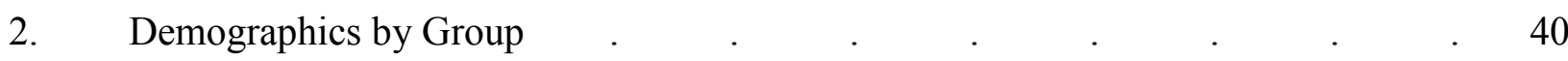

3. Median Ratio of Subjective to Objective Number of Postpartum Awakenings by Week .

4. Correlations between Number of Awakenings and Performance Variables at Weeks 2 and 12 


\section{LIST OF FIGURES}

1. Flowchart of algorithm coding raw actigraphy data as Sleep or Wake

2. Frequency histogram of controls' awakening duration threshold values in seconds with a normal curve

3. Histogram of the ratio of subjective to objective number of awakenings at week 12

4. Mean objective number of awakenings per night with standard error bars measured during postpartum week 2 and 12

5. Mean objective duration of awakenings per night with

standard error bars measured during postpartum week 2 and 12

6. Objective number of awakenings per night at postpartum week 2 and 12 with separate lines by feeding method

7. PVT lapses with error bars at postpartum week 2 and 12 with separate lines by feeding method .

8. PVT median reaction time at postpartum weeks 2 and 12 with separate lines by feeding method

9. Histogram of median reaction time at week 2 


\section{LIST OF APPENDICES}
A. Number of Awakenings PDA Prompt
52
B. Duration of Awakenings PDA Prompt
53
C. Psychomotor Vigilance Test .
54
D. Screening Dialogue and CES-D
E. Referral List . 


\section{Introduction}

Sleep disturbance is a common problem that causes a variety of negative physiological and psychological effects, the most notable of which are reductions in cognitive abilities such as attention, reaction time, and working memory (Banks \& Dinges, 2007; Bonnet, 2005). During sleep, new memory creation is suspended (Perlis, Smith, Orff, Andrews \& Giles, 2001) and complex memory processing occurs (Ji \& Wilson, 2007). This study examined new memory formation during nocturnal awakenings among postpartum mothers, who regularly experience sleep disturbance in the form of multiple awakenings from sleep, generally for the purpose of infant caretaking. This study evaluated how these awakenings change between the initial and later postpartum period, as well as possible associations between feeding methods and neurocognitive performance. The data used for this study came from an existing database, which was created for the purposes of analyzing postpartum sleep fragmentation and daytime functioning among first-time mothers.

The introduction section of this paper has been organized as follows. Normal adult sleep and types of sleep disturbance are described, as well as psychomotor vigilance testing, the outcome measure used to assess neurocognitive performance in the present study. Memory consolidation is discussed in terms of sleep and the amnesic properties of the sleep onset period. Then, the sleep of postpartum mothers will be described, as well as the value of this population for the study of sleep. Infant feeding methods will be presented in terms of their relation to maternal sleep variables.

\section{Normal Adult Sleep}

The normal adult sleep cycle consists of several predictably recurring physiological and behavioral state changes, which last a total of approximately 90-110 minutes. These states are 
categorized into non-rapid-eye-movement (NREM) sleep and rapid eye movement (REM) sleep. In a normal transition from wake to sleep, the individual will consecutively progress through each of the NREM sleep stages: N1, N2, and N3 (also called Slow Wave Sleep [SWS]). Each of these stages is associated with progressively higher arousal thresholds, making N1 the lightest stage of sleep and N3 the deepest sleep stage. After N3, there is a transition back through N2 and then into REM sleep, an active dreaming stage characterized by rapid eye movement. When a cycle completes, the normal individual will briefly awaken, then start another cycle. Throughout a normal 8-hour consolidated sleep period, 4 or 5 complete cycles will occur, meaning that adults will naturally awaken 4 or 5 times per night. Sleep cycles lengthen throughout the night with SWS making up a greater proportion in the earlier cycles, and REM increasing in duration across each successive cycle (Carskadon \& Dement, 2011).

\section{Sleep Disturbance}

Sleep disturbances are common and generally occur due to a reduction in overall sleep duration (sleep deprivation) or due to an interruption to the sleep cycles (sleep fragmentation). Shapiro and Dement (1993) estimated that sleep problems cost the United States \$16 trillion every year. These costs come in the form of industrial accidents, motor vehicle accidents, reduced work performance and medical costs (Connor, Whitlock, Norman, \& Jackson, 2001; Landrigan et al., 2004; Leger, 1994; Lockley et al., 2004; Rosekind, 2005). Costs to the individual can include deficits to immune function, attention, reaction time, and memory, as well as increased irritability and increased appetite (Orzel-Gryglewska, 2010).

The negative consequences of sleep deprivation and sleep fragmentation are similar, each causing a host of physiological and psychological problems (Downey \& Bonnet, 1987;

Stepanski, 2002). The specific effects of fragmentation, though, depend on the individual stages 
being interrupted. Slow wave sleep is considered regenerative, and its selective restriction is associated with reductions in immune function and cell repair. Selective REM deprivation is associated with the negative neurocognitive effects of sleep disturbance on attention, reaction time, and memory function (Orzel-Gryglewska, 2010).

One of the most widely used measures of performance deficits due to sleepiness is the psychomotor vigilance test, a measure shown to be reliable, valid, and highly sensitive to sleep disturbance (Lim \& Dinges, 2008). During each test, participants press a button in response to the presentation of stimuli with variable inter-stimulus intervals. Sleep disturbance has been shown to cause increased median reaction time, increased variability in responding, increased number of false starts and increased number of lapses (delays in responding of $500 \mathrm{~ms}$ or greater). These lapses are considered to be 'micro-sleeps' and are highly predictive of sleepiness, as well as being directly applicable to practical safety concerns such as falling asleep while driving (Lim $\&$ Dinges, 2008). For these reasons, median reaction time and number of lapses on the psychomotor vigilance test were used as our measures of neurocognitive performance in this study.

\section{Sleep and Memory}

According to the activation/synthesis model, memory traces are formed during the day and stored temporarily in the hippocampus. During SWS, the neural networks that were active during the creation of those traces are reactivated and undergo some minor consolidation across the cortex. During REM sleep, these traces are further consolidated and converted to long-term memories by co-activation and comparison with pre-existing long-term memories. A variety of work in both humans and animals supports this model (Cartwright, 2004; Ji \& Wilson, 2007; Pennartz, Uylings, Barnes, \& McNaughton, 2002; Takehara-Nishiuchi \& McNaughton, 2010). 
While this memory consolidation is occurring, however, the ability to form new memories of external events is almost completely eliminated (Perlis, et al., 2001); though, a recent study by Fifer (2010) found that delay eye-blink conditioning can be shown in infants during sleep. Also, some basic memory processing is occurring, as evinced by recall of dreams during abrupt awakenings during REM sleep (Shapiro, Goodenough \& Gryler, 1973).

This amnesic effect of sleep also partially extends into the sleep/wake transition (the several minutes immediately prior to sleep onset). Individuals do not recall falling asleep, and there is some evidence that new memory processing gradually reduces during this transition (Portnoff, Baekeland, Goodenough, Karacan, \& Shapiro, 1966; Wyatt, Bootzin, Anthony, \& Bazant, 1994). Also, memories are rarely formed for the brief awakenings which normally occur between every sleep cycle (Goodenough, Sapan \& Cohen, 1971). For each of these periods (sleep onset and nocturnal awakenings), a rule of thumb has arisen that approximately 5 minutes of continued wakefulness are required after a stimulus is presented in order for later recall of that stimulus. The few studies that have directly examined this phenomenon vary on the duration necessary for recall (Bonnet, 1983; Goodenough, Sapan \& Cohen, 1971; Portnoff, Baekland, Goodenough, Karacan \& Shapiro, 1966; Wyatt, Bootzin, Anthony, \& Bazant, 1994; Wyatt, Bootzin, Allen, \& Anthony, 1997).

Initial research into this phenomenon of sleep transition amnesia focused primarily on memory during awakenings rather than during sleep onset. In 1966, Portnoff, Baekland, Goodenough, Karacan and Shapiro found that morning retention for verbal materials presented to participants during forced awakenings was significantly better if followed by a period of enforced wakefulness. The authors concluded from this that NREM sleep interferes with the consolidation of memory traces. In a series of experiments by Goodenough, Sapan and Cohen 
(1971), participants kept awake for 5 minutes completing a psychomotor task during a forced nocturnal awakening had increased morning recall of words presented before the task compared to those who were allowed to immediately return to sleep. However, the results also showed that when the psychomotor task was presented as a game rather than as a test, this difference between groups disappeared. The experimenters also noticed that participants were notably less aroused during the game trials than during the test trials, and concluded that arousal level may be as important as duration of wakefulness.

Bonnet (1983) disagreed with the findings of previous studies. He found no difference in participants' morning recall when given 8 minutes of enforced wakefulness after awakening rather than immediately returning to sleep. Instead, he found that participants awakened from slow wave sleep (N3) performed much worse than those awakened from N2, not only on morning recall, but also on a short-term memory task given during the awakening. However, in this study the short-term memory task itself took 8 minutes, so the actual awakening durations being compared in this study were 8 minutes and 16 minutes, well beyond the 5 minutes hypothesized by previous work. Considering that there were still differences between those participants awakened from N3 and N2, it could be the case that previous sleep stage is as important as duration of wakefulness or more so. In following with the conclusions of Goodenough, Sapan and Cohen (1971), it may also be that the elevated arousal threshold of N3 results in lower subsequent arousal during the tasks compared to N2.

Over a decade later, Wyatt et al. (1994) examined memory for stimuli presented during sleep onset by verbally presenting a word pair each minute as the participants fell asleep. They were then either awakened after 30 seconds or 10 minutes of EEG-defined sleep, and were given a free recall task and a recognition task for the word pairs presented. The free recall task 
consisted of participants saying aloud to the researcher all word pairs that they could remember being presented. The recognition task consisted of verbal presentation of word pairs that either were or were not presented previously and the participants were asked to identify word pairs that they remembered being presented. In the 10 minute condition, the authors found almost no free recall for word pairs presented to participants during the 3 minutes prior to sleep onset and a significant lowering of recognition only for words presented during the last minute prior to sleep. Participants in the 30 second condition showed none of these differences based on minute prior to sleep onset for the recall and recognition tasks. However, both conditions did show an overall decrease in recall and recognition scores compared to an awake control group. A later replication and expansion on this work (Wyatt, Bootzin, Allen, \& Anthony, 1997) found similar results with significant impairment in free recall for words presented during the 4 minutes prior to sleep onset, but no significant differences for recognition scores based on minute presented prior to sleep. This failure to replicate the previous findings regarding recognition was likely due to differences in experimental design; the recognition task of the original study included words from the 8 minutes prior to sleep onset whereas the replication included words from only 3 minutes prior to onset.

Except for Bonnet (1983), all of the studies investigating memory during the sleep/wake transition indicate the importance of duration of awakening for morning recall, though the duration itself is disputed. Experimental design issues call into question Bonnet's conclusions on duration of awakenings, but his work does highlight the potential importance of sleep stage awakened from and supports the earlier observation by Goodenough, Sapan and Cohen (1971) that arousal level may also be important. 
Of these studies, however, none occur in a natural sleep setting, and all had artificial reasons for awakening and maintaining wakefulness, such as memorizing words, completing psychomotor tasks, etc. Considering the variety of results in experimental settings (Table 1), it would follow that results may be very different if these awakenings took place in a natural setting, were non-experimental (such as for infant feedings) and the tasks were routine.

\section{Postpartum Sleep}

One population that experiences regular nocturnal awakenings of varying duration is new parents. Early parenthood is associated with sleep disturbance, and recent evidence has shown that though new mothers are getting more total sleep time than previously thought, their sleep is highly fragmented, with multiple awakenings throughout the night (Montgomery-Downs, Insana, Clegg-Kraynok, \& Mancini, 2010). This fragmentation is determined in great part by the needs of the infant, who exhibits poly-phasic sleep and a need for regular feedings throughout the night (Carskadon \& Dement, 2011). Infant sleep and feeding patterns determine the mother's sleep patterns (Thomas \& Foreman, 2005) and over time, as the infant's sleep consolidates, the mother may be able to return to sleeping through the night, or at least to a less fragmented pattern. If we define "sleeping through the night" as sleeping from 10pm to 6am, most infants first begin to do so at five months of age, with rapid consolidation occurring during the first four months (Henderson, France, Owens, \& Blampied, 2010).

The current study evaluated memory for nocturnal awakenings in postpartum mothers and characterizes how these awakenings change during the rapid consolidation of infant sleep during the early postpartum period. 


\section{Infant Feeding Methods}

A major reason for nocturnal awakenings throughout the early postpartum period is for infant feeding. Breastfeeding has numerous benefits for both the infant and the mother (Wu \& Chen, 2009), and is strongly recommended by the American Academy of Pediatrics: "breastfeeding ensures the best possible health as well as the best developmental and psychosocial outcomes for the infant" (p. 501, 2005). However, mothers worry about how breastfeeding will affect their own sleep. Recent work by Montgomery-Downs, Clawges, and Santy (2010) found no differences between women using different feeding methods on the objective measures of total sleep time, sleep efficiency, or sleep fragmentation, nor on the subjective measures of number of awakenings, total wake time, sleep quality, sleepiness or fatigue during postpartum weeks 2 through 12 . Thus, current research suggests that a new mother's choice of feeding method will not necessarily affect her sleep.

However, prolactin, a protein necessary for breast milk production, peaks in concentration during the night (Ross, 2005). Prolactin levels further increase during and after breastfeeding (Kent, 2007). In non-human animals, injection of prolactin or vasoactive intestinal peptide (which stimulates increased release of endogenous prolactin) each increase REM sleep and injection of a prolactin antiserum decreases REM sleep (Roky, Obál, Valatx, Bredow, Fang, Pagano \& Krueger, 1995; Stieger, 2003). In humans, intravenous injection of vasoactive intestinal peptide also increases subsequent REM sleep; however, direct injections of prolactin or prolactin antiserum have not been studied (Roky, Obál, Valatx, Bredow, Fang, Pagano \& Krueger, 1995). With these facts in mind, it would follow that continued nocturnal breastfeeding could result in more REM sleep compared to other feeding methods, due to increased prolactin levels, despite no observable differences in subjective and objective measures of sleep. Since 
REM is associated with the neurocognitive benefits of sleep, there may yet be unmeasured differences in neurocognitive performance between women who use different feeding methods. Breastfeeding may have a compensatory effect against decreased sleep efficiency by increasing the amount of time spent in REM. A test of neurocognitive performance, such as psychomotor vigilance, could reveal evidence for this effect despite a lack of differences in other sleep measures. Thus, in addition to evaluating memory for awakenings in the current study, the association between feeding methods and neurocognitive performance also was evaluated.

\section{Statement of the Problem}

Although 5 minutes of wake is the accepted 'rule of thumb' in the sleep field for the amount of time necessary to encode new memories, few studies have directly examined this phenomenon or how it may be affected by sleep disturbance. None have done so among normative, sleep disturbed samples using a field-based protocol. Postpartum mothers are an excellent population for studying this phenomenon due to their regular, natural awakenings as well as regular sleep disturbance. Remembering these awakenings could be important during the postpartum period as the mothers would have a better understanding of their own sleep fragmentation. Also, inability to accurately recall awakenings may be an indicator of neurocognitive disturbance during the postpartum period.

We know that postpartum maternal sleep fragmentation decreases over time, but we do not know the details of how this fragmentation is changing. If we did, new parents could better plan for shared responsibility of infant needs in order to reduce maternal sleep fragmentation. 
Finally, it is important to know if breastfeeding is associated with reduced neurocognitive deficits during this period of sleep disturbance. If so, this would show yet another benefit of breastfeeding and one that could result in a safer, healthier postpartum period.

\section{Objective/Purpose}

This study was exploratory in regards to morning recall of nocturnal awakenings due to the lack of research on memory for normal, naturally occurring awakenings. Accuracy of maternal morning recall of awakenings was investigated as compared to a control group of women who have never had children. This study was also exploratory in regards to characterizing the number and duration of early and late postpartum awakenings. No previous research has directly measured awakenings based on duration. Finally, this study also evaluated objective differences on neurocognitive performance between women using different infant feeding methods.

\section{Research Questions and Hypotheses}

Research Questions 1-3 are exploratory. RQ1: What is the minimum length of a nocturnal awakening necessary for morning recall in controls? Compared to controls, how accurate is postpartum memory for nocturnal awakenings during postpartum weeks 2 and 12?

For this research question, memory in the morning for each previous night's awakenings (measured by actigraphy) in nulliparous controls was examined and compared to those data of new mothers at postpartum weeks 2 and 12. It was expected that the accuracy of postpartum recall for awakenings would increase between weeks 2 and 12 . 
RQ2: How will the number and length of nocturnal awakenings change from postpartum week 2 to 12 ?

For this research question, early and late postpartum sleep fragmentation was characterized by examining the objectively measured number and duration of awakenings (via actigraphy) during nocturnal sleep periods during postpartum week 2 and 12. It was expected that the overall number and length of awakenings would decrease between postpartum weeks 2 and 12 .

RQ3: Are there differences in neurocognitive performance based on objective number of awakenings during postpartum weeks 2 and 12 ?

For this research question, objective differences in median reaction time and number of PVT lapses were evaluated based on objectively measured number of awakenings (via actigraphy) during postpartum weeks 2 and 12. It was expected that participants with more awakenings would have poorer slower reaction times and more lapses on the PVT regardless of week.

RQ4: Are there differences in objective number of awakenings between women who breast feed and women who formula feed during postpartum weeks 2 and 12 ?

H1: During postpartum weeks 2 and 12, there will be no differences in objectivelydetermined number of awakenings based on feeding method. Previous work has shown no differences in total sleep time, sleep efficiency, sleep fragmentation, or subjective number of awakenings based on feeding method during weeks 2 and 12 postpartum (Montgomery-Downs, et al., 2010). 
RQ5: Are there differences in neurocognitive performance between women who breast feed and women who formula feed during postpartum weeks 2 and 12?

H1: During postpartum week 2, there will be no differences in median reaction time or number of PVT lapses based on feeding method. The proposed compensatory effect of breastfeeding would be cumulative, and thus, is not likely to be detectable earlier in the postpartum period.

H2: During postpartum week 12, breast-feeding mothers will have increased faster median reaction times and fewer PVT lapses compared to formula-feeding mothers. Due to the hypothesized compensatory effect of prolactin release during breast-feeding, breast-feeding mothers will have more REM sleep and thus, improved reaction times and fewer lapses. 


\section{Method}

\section{Participants}

This study used preexisting data from a larger study of primiparous mothers in Morgantown, WV and the surrounding region. Demographic data for this study are summarized in Table 2. Participants differed significantly from controls on education, income, and marital status. Differences in income were mainly due to most controls being unmarried and thus lacking shared income. Control participants followed the same procedures as the postpartum women.

Measures

Actigraphy. Participants continuously wore a Mini Mitter Actiwatch-64 (AW64) actigraph on their non-dominant wrist, except in situations where the device might get wet. The wristwatch-sized actigraph is a non-intrusive device containing an accelerometer that constantly records movement direction and movement intensity data over time. Along with sleep logs recorded on a Personal Digital Assistant (PDA), these data are used to quantify participants' sleep/wake patterns. Actigraphy has been extensively validated among adults as a method for study of sleep/wake patterns (Benson, Friedman, Noda, Wicks, Wakabayashi, \& Yesavage, 2004; King, Jaffre, Morrish, Shneerson \& Smith, 2005; Sadeh, Sharkey \& Carskadon, 1994). The actigraph could be removed at any time (for bathing, washing dishes, etc.), but participants were instructed to record removal/re-attachment on a Personal Digital Assistant. Actigraphs were collected and replaced with new ones during weekly home visits by the researchers.

Personal Digital Assistant (PDA). Participants were also assigned a Palm Zire 72 handheld computer programmed specifically for this study. The program included a sleep diary, 
actigraph on/off diary, and the psychomotor vigilance test. The program allowed participants to record when they went to bed or when they woke up, when they removed or reattached the actigraph, and the number and duration of awakenings in the previous night. PDA's were collected and replaced with new ones during weekly visits by the researchers.

Self-report of Awakenings. Each morning participants were prompted to answer questions on the PDA regarding the previous night's awakenings. The first prompt was "How many times do you think you woke up last night?" and could be answered by clicking the arrows of a counter box and pressing "Next" (See Appendix A). The second prompt was "Please indicate how long you were awake last night (total)." and could also be answered by clicking the arrows of a counter box and pressing "Next" (See Appendix B).

Psychomotor Vigilance Test (PVT). The PVT program used was similar to Ambulatory Monitoring Inc.'s palm-based React Software, wherein participants attempt to respond as fast as possible to the presentation of a stimulus (a bull's-eye) by pressing a button on the PDA, which then removes the stimulus and begins another trial (See Appendix C). Stimuli were presented with variable inter-stimulus intervals, and testing continued until 5 minutes had elapsed. For each test, reaction time was recorded for each trial. Participants were asked to take the PVT each morning within two hours of awakening and before consuming any caffeine so as to prevent possible alerting effects due to circadian timing or caffeine, respectively (Lim \& Dinges, 2008).

Feeding Method. After their participation in the study was complete, each participant was asked during a telephone interview to describe their feeding method during the first week and was categorized as exclusively breast-feeding, exclusively formula-feeding, or any combination of both methods. Those in the breast-feeding or combination group were then asked if they had switched methods during the study and at which week the switch had occurred. 


\section{Procedure}

Participants were recruited prenatally via advertisements in a variety of public postings and publications to facilitate recruitment of a range of participants, including low-income and minority women.

An initial phone interview (See Appendix D) screened for depression as an exclusion criterion using the Center for Epidemiologic Studies Depression Scale (CES-D) developed by Radloff (1977). If the potential participant had a CES-D score $\geq 16$, they were excluded from the study and given information on community resources for depression (See Appendix E). Then, a prenatal home visit allowed for administration of informed consent and Health Insurance Portability and Accountability Act (HIPAA) authorization, as well as training on use of the actigraph and PDA. Participation began at the end of Week 1 postpartum and continued until the end of Week 13. Participation of controls lasted for one week.

Each morning within two hours of awakening, participants would record waking time on their PDA and the number and overall duration of awakenings. Then they would complete the PVT. At night, participants would record when they were going to bed. During weekly visits in the home, researchers would collect and replace PDAs and actigraphs.

Participants were compensated each week with a small gift or book for the infant, as well as $\$ 336$ at completion of the 12 week study period. Participants who dropped out of the study early were compensated $\$ 14$ per week of participation.

\section{Data Analysis}

PVT Latency. In order to verify that participants were performing the PVT within 2 hours of awakening as requested, PVT latency was calculated as the time between objectively determined awakening (via actigraphy) and beginning the PVT. In the control group, $11.4 \%$ of 
all PVT trials were not initiated within 2 hours of awakening. However, average participant PVT latencies after awakening were not significantly associated with median reaction times, $r(25)=$ $.261, \mathrm{p}=.189$, or number of lapses, $\mathrm{r}(24)=-.336, \mathrm{p}=.094$.

In the postpartum group at weeks 2 and $12,15 \%$ and $25.5 \%$ of all PVT tasks were not initiated within 2 hours of awakening, respectively. However, at week 2, average participant PVT latencies after awakening were not significantly associated with median reaction time, $r(40)$ $=-.016, \mathrm{p}=.922$, or number of lapses, $\mathrm{r}(40)=-.055, \mathrm{p}=.729$. At week 12, average participant PVT latencies after awakening were also not significantly associated with median reaction time, $\mathrm{r}(40)$ $=.120, \mathrm{p}=.451$, or number of lapses, $\mathrm{r}(40)=.143, \mathrm{p}=.367$. Therefore, PVT latency was not significantly associated with PVT performance at postpartum week 2 or week 12, or among control participants and all data were retained.

Awakening Coding. Participant awakenings were identified by an algorithm applied to raw actigraphy data (epoch by epoch). This algorithm required 2 minutes of inactivity ( 8 consecutive epochs with no activity) to begin coding epochs as sleep. Consistent with polysomnograpy (PSG), epochs were coded as wake only when 30 seconds of activity (2 consecutive epochs of activity) was detected (Figure 1). The number of awakenings at each duration (e.g. $\geq 30 \mathrm{sec} ; \geq 45 \mathrm{sec} ; \geq 60 \mathrm{sec} ; \geq 75 \mathrm{sec}$ ) were then totaled for each participant within each week.

Feeding Method Coding. Among the final sample only three participants fell into the feeding method category of exclusively formula-feeding during both weeks 2 and 12 . To increase statistical power, participants who switched feeding methods very early in the study (at week 3) were recoded as their final method, and participants who switched feeding methods very late in the study (at week 11) were recoded as their initial method. At week 3, two participants 
reported switching from using both methods to exclusively formula-feeding beginning. These two participants (who only partially breastfed for the first week of the study) were recoded as exclusively formula-feeding. At week 11, no participants reported switching methods.

Statistical Analyses. All statistical tests were performed using PASW Statistics, Release 18.0.2 (April 2010). For all analyses, a p-value of less than .05 was required for significance and Cohen's d was calculated to determine effect size. Microsoft Excel 2010 was used for algorithm processing and for creation of bar and line graphs.

Research Question 1: Output from the actigraph algorithm was used to establish individual awakening duration threshold values for each control participant. This threshold value was calculated as the duration of an awakening (e.g. $\geq 30 \mathrm{sec} ; \geq 45 \mathrm{sec}$ ) at which the total objective number of awakenings (via the actigraph algorithm) matched the total subjective number of awakenings (via self-report).

The median threshold of controls was calculated and applied to data from the postpartum women to determine the number of objective awakenings that each could be expected to selfreport based on the control-determined threshold. These data were then compared to the actual self-reported number of awakenings in order to determine recall accuracy. A ratio of subjective to objective number of awakenings was then calculated for each participant at postpartum weeks 2 and 12.

Research Question 2: In order to compare numbers of awakenings between postpartum weeks, a repeated-measures ANOVA was performed on participants between postpartum week 2 and 12 with objectively measured number of awakenings (via actigraphy) as the outcome measure. In order to compare durations of awakenings between postpartum weeks, another repeated-measures ANOVA was then performed on participants between postpartum week 2 and 
12 but with objectively measured durations of awakenings (via actigraphy) as the outcome measure.

Research Question 3: In order to analyze potential relations between characteristics of awakenings and neurocognitive performance measures, bivariate correlations were calculated for average number of awakenings and average total duration of awakenings per night at each postpartum week, PVT lapses, and PVT median reaction time.

Research Question 4: In order to determine if there are differences in number of awakenings between women who breast feed and women who formula feed during postpartum weeks 2 and 12, participants were separated into those exclusively breastfeeding and those exclusively formula-feeding, then a $2 \times 2$ repeated measures ANOVA (feeding method by postpartum week) was performed with objectively measured number of awakenings (via actigraphy) as the outcome measure.

Research Question 5: In order to determine if there were differences in neurocognitive performance between women who breast feed and women who formula feed during postpartum weeks 2 and 12, participants were again separated into those exclusively breastfeeding and those exclusively formula-feeding, then a $2 \times 2$ repeated-measures ANOVA (feeding method by postpartum week) was performed with PVT lapses as the outcome measure. Another 2x2 repeated-measures ANOVA (feeding method by postpartum week) was performed with median reaction time as the outcome measure. 


\section{Results}

\section{Missing Data}

Twenty-seven out of 71 potential postpartum participants from the existing data were excluded from the analyses due to having fewer than 4 nights of available actigraphy or PDA data at either postpartum week 2 or week 12. These missing data were due to equipment malfunction or protocol non-adherence. This resulted in a final $\mathrm{N}$ of 44 .

Of these, three additional participants did not have feeding method data during weeks 2 or 12 and were not included in the analyses for RQ4 and RQ5.

Four participants had fewer than 4 days of completed PVT tasks during week 2 or 12 and were not included in the analyses for RQ3 and RQ5.

RQ1: What is the minimum length of a nocturnal awakening necessary for morning recall in controls?

Output from the actigraph algorithm was used to establish individual awakening duration threshold values for each control participant. This threshold value was calculated as the duration of an awakening (e.g. $\geq 30 \mathrm{sec} ; \geq 45 \mathrm{sec}$ ) at which the total objective number of awakenings (via the actigraph algorithm) matched the total subjective number of awakenings (via self-report). The objective thresholds of the control participants for subjective recall ranged from $45 \mathrm{~s}$ to $600 \mathrm{~s}$ with a mean of $288.9 \mathrm{~s}(\mathrm{SD} \pm 130.3)$ and a median of $270 \mathrm{~s}$. Figure 2 illustrates that thresholds were also normally distributed (skewness $[\mathrm{SE}]=.43[.45]$, kurtosis $[\mathrm{SE}]=.23[.87]$ ). The median threshold was used rather than the mean for a conceptual reason: because PSG epochs are scored using 30-second epochs.

Compared to controls, how accurate is postpartum memory for nocturnal awakenings during postpartum weeks 2 and 12? 
The median threshold of 270s was applied to data from the postpartum women to determine the number of objective awakenings that each could be expected to self-report based on the control-determined threshold. These data were then compared to the actual self-reported number of awakenings in order to determine recall accuracy. A ratio of subjective to objective number of awakenings was then calculated for each participant at postpartum weeks 2 and 12 .

After calculating participants' ratios, a single outlier was found at week 12 who was 5.4 standard deviations from the group mean. This participant had a normal number of subjective awakenings (19) as well as a normal number of objective awakenings (2), however, this combination lead to an outlier ratio of 9.5 (Figure 3).

After thorough investigation of the participant's raw data, this outlier was determined not to be due to participant error or equipment failure, and removal could not be justified due to similarly extreme ratios measured for control participants with low awakening duration thresholds. Medians were determined to be better measures of central tendency in this case and are presented in Table 3 along with median subjective and objective number of awakenings per night at weeks 2 and 12 .

At week 2 postpartum women reported a median of $52.4 \%$ of awakenings and at week 12 they reported a median of $79.1 \%$ of awakenings. A Related Samples Wilcoxon Signed Rank Test for the difference between the medians found that this increase in accuracy between weeks (26.7\%) was statistically significant, $p<.01$.

Participants underreported awakenings to a greater degree during week 2 than during week 12. Thus, the accuracy of postpartum women for reporting awakenings increased between postpartum week 2 and week 12 . 
RQ2: How will the number and length of nocturnal awakenings change from postpartum week 2 to 12 ?

A repeated-measures ANOVA was performed with postpartum week as the independent variable and mean number of objectively measured awakenings per night (via actigraphy using 270s-threshold-identified awakenings) as the dependent variable. Participants averaged 5.7 awakenings per night during week 2 and 3.2 awakenings per night during week 12 for a statistically significant mean reduction of 2.5 awakenings per night, $\mathrm{F}(1,43)=49.7, p<.001$, $d=1.3$ (Figure 4).

Another repeated-measures ANOVA was performed with postpartum week as the independent variable and mean total duration of objectively measured awakenings per night (via actigraphy using 270s-threshold-identified awakenings) as the dependent variable. Results showed a significant effect of postpartum week, $\mathrm{F}(1,43)=58.6, p<.001, d=1.2$. Participants averaged 78.8 minutes awake per night during week 2 and 38.9 minutes awake per night during week 12 for a mean reduction of 39.9 minutes awake per night (Figure 5).

RQ3: Are there differences in neurocognitive performance based on objective number of awakenings during postpartum weeks 2 and 12 ?

Bivariate correlations were calculated between both neurocognitive performance measures (PVT lapses, PVT median reaction time) and average number of awakenings per night at each postpartum week (Table 4). As expected, PVT measures were tightly intercorrelated at each week and between weeks. At week 2, objectively measured number of awakenings was not significantly associated with number of PVT lapses $(\mathrm{r}=-.036, p=.820)$ or PVT median reaction time $(\mathrm{r}=-.058, p=.715)$ At week 12 , number of awakenings was also not significantly associated with number of PVT lapses $(\mathrm{r}=-.055, p=.729)$ or PVT median reaction time $(\mathrm{r}=-.149, p=.345)$. 
An unexpected finding was that duration of awakenings at week 2 was significantly related to week 12 PVT lapses $(\mathrm{r}=.430)$ and PVT median reaction time $(\mathrm{r}=.353)$.

RQ4: Are there differences in number of awakenings between women who breast feed and women who formula feed during postpartum weeks 2 and 12 ?

H1: During postpartum weeks 2 and 12, there will be no differences in objectively determined number of awakenings based on feeding method.

Participants were separated into those coded as exclusively breastfeeding and those coded as exclusively formula-feeding, then a $2 \times 2$ repeated-measures ANOVA (feeding method by postpartum week) was performed with objectively measured number of awakenings per night (via actigraphy) as the outcome measure. Consistent with RQ2, results showed a significant main effect of week, $\mathrm{F}(1,17)=18.9, p<.001, d=1.1$, with $42.3 \%$ fewer awakenings per night occurring during week 12 (Figure 6). Hypothesis 1 was supported in that there was no significant main effect of feeding method, $\mathrm{F}(1,17)=.34, p=.568$. There was also no significant interaction effect of feeding method by postpartum week, $\mathrm{F}(1,17)=.47, p=.503$. RQ5: Are there differences in neurocognitive performance between women who breast feed and women who formula feed during postpartum weeks 2 and 12 ?

H1: During postpartum week 2, there will be no differences in neurocognitive performance based on feeding method.

H2: During postpartum week 12, breast-feeding mothers will have increased neurocognitive performance compared to formula-feeding mothers.

Participants were separated into those exclusively breastfeeding and those exclusively formula-feeding at both weeks, then a 2x2 repeated-measures ANOVA (feeding method by postpartum week) was performed with PVT lapses as the outcome measure. Results showed a 
significant main effect of week, $\mathrm{F}(1,17)=10.8, \mathrm{p}<.005, d=.50$, with an average of 3.1 more lapses occurring each night during week 12 (Figure 7). There was no significant main effect of feeding method, $F(1,17)=2.6, p=.125$. There was also no significant interaction effect of feeding method by postpartum week, $\mathrm{F}(1,17)=2.6, \mathrm{p}=.128$.

Another 2x2 repeated-measures ANOVA (feeding method by postpartum week) was performed with median reaction time as the outcome measure. Results showed a significant main effect of week, $\mathrm{F}(1,17)=4.73, \mathrm{p}<.05, d=.27$, with an average of $22 \mathrm{~ms}$ slower median reaction times occurring during week 12 (Figure 8). There was also a significant main effect of feeding method, $\mathrm{F}(1,17)=4.54, \mathrm{p}<.05, d=.87$, with those formula-feeding having an average of 70.6ms slower reaction times than those breastfeeding. There was not a significant interaction effect of feeding method by postpartum week, $\mathrm{F}(1,17)=.59, \mathrm{p}=.453$.

H1 was partially supported in that there were no differences on PVT lapses by feeding method at week 2 , however exclusively breastfeeding mothers did have improved median reaction time compared to exclusively formula-feeding mothers at week 2 .

H2 was also partially supported in that at week 12 exclusively breastfeeding mothers did have improved median reaction time compared to exclusively formula-feeding mothers, however there were no differences on PVT lapses by feeding method.

However, one participant was found to be an outlier at week 2 with an average median reaction time of $623 \mathrm{~ms}$ (4.1 standard deviations from the mean, Figure 9).

Careful investigation of the raw data determined that this outlier was not due to human error. Further checking revealed that the participant had consistently higher than average median reaction times and PVT lapses throughout the study. The participant was not removed from 
analyses. However, because there would be significant effects if this participant were removed, those effects should be mentioned.

The relation between duration of awakenings at week 2 and number of awakenings at week 12 became significant, $\mathrm{r}=.323, p<.05$. The relation between duration of awakenings at week 2 and median reaction time at week 12 was no longer found to be significant, $\mathrm{r}=.246$, $p=.120$. The significant main effect of feeding method on median reaction time in RQ5 was no longer found to be significant, $\mathrm{F}(1,16)=2.8, p=.113, d=.73$. 


\section{Discussion}

This study investigated memory and neurocognitive performance variables as they relate to sleep during the postpartum period. The main questions involved memory for brief awakenings in controls and postpartum women, as well as potential differences in neurocognitive performance between postpartum women who use different feeding methods. This section will discuss these questions and what results from this study may mean, as well as how this study fits into the current research literature. Limitations and future directions will also be discussed.

\section{Memory for Brief Awakenings}

The first research question explored self-reported recall of brief awakenings in controls. The current 5 minute rule of thumb for recalling awakenings suggests that the duration of an awakening is important for morning recall (Goodenough, Sapan \& Cohen, 1971). By matching participants' recalled awakenings with their actual awakenings sorted by duration, a median threshold of 270 seconds was found with normal variability. This 4 minute 30 second threshold closely coincides with previous work to suggest that approximately 5 minutes of wakefulness may be necessary for morning recall (Portnoff, Baekland, Goodenough, Karacan \& Shapiro, 1966; Wyatt, Bootzin, Anthony, \& Bazant, 1994; Wyatt, Bootzin, Allen, \& Anthony, 1997). This study represents the first direct investigation of memory during natural awakenings in the home.

Thresholds ranged from 45 seconds to 10 minutes, indicating large variability even among controls. This variability could just represent a natural variability in durations of wakefulness necessary for recall, but may also indicate that other variables affect this threshold, such as: stage of from which the individual awoke (Bonnet, 1983), amount of sleep between awakening and recall (Wyatt et al., 1994; Wyatt, Bootzin, Allen, \& Anthony, 1997), or arousal 
level during awakenings (Goodenough, Sapan \& Cohen, 1971). This variability may also be a factor in a condition known as Sleep State Misperception (SSM), wherein individuals regularly and inaccurately underestimate sleep or overestimate awakenings (Edinger \& Krystal, 2003). These individuals may have very low awakening duration thresholds necessary for recall. Postpartum mothers' accuracy of recall was assessed against this 270 second threshold. Postpartum women underestimated the number of awakenings at both week 2 (52\% accurate) and week 12 (79\% accurate), however, accuracy did significantly increase between week 2 and week 12 (27\% increase).

These findings would indicate memory for awakenings is initially impaired during the postpartum period, but improved significantly by week 12 . These findings coincide with work showing that initial impairment of verbal, semantic, and working memory during the early postpartum period improves over time (Buckwalter, Buckwalter, Bluestein, and Stanczyk, 2001). With the increasing sleep efficiency and decreased sleep fragmentation found across the postpartum period for women in this dataset (Montgomery-Downs, Insana, Clegg-Kraynok, \& Mancini, 2010), it is not surprising that cognitive functions would recover over time. Another potential explanation for this increase in accuracy is that with fewer awakenings occurring each night during week 12 , each of the awakenings was more salient and therefore easier to recall.

\section{Characteristics of Postpartum Awakenings}

The second research question aimed to characterize awakenings during the postpartum period. Participants had a higher average number of awakenings per night during week 2 (5.7) compared to week 12 (3.2). Participants also had more than double the average total duration of awakenings per night during week 2 (78.8 minutes) compared to week 12 (38.9 minutes). Across the first 12 postpartum weeks, these new mothers were awakening less often with less 
total wake time after sleep onset. Since infant sleep and feeding patterns determine the mother's sleep patterns (Thomas \& Foreman, 2005) and infant sleep rapidly consolidates over the first 16 postpartum weeks (Henderson, France, Owens, \& Blampied, 2010), this could result in fewer maternal awakenings during this period. These findings also coincide with previous work on this dataset, which found decreased sleep fragmentation and increased sleep efficiency across the postpartum period (Montgomery-Downs, Insana, Clegg-Kraynok, \& Mancini, 2010). Also, it is important to note that these values did not include brief arousals which were shorter than the 4 minute, 30 second threshold for recall.

Research question three dealt with potential associations between the measured characteristics of awakenings (average number per night and average total duration per night) and measures of neurocognitive performance (average number of PVT lapses and median reaction time). As expected, measures of neurocognitive performance were highly positively correlated within and between postpartum weeks (Table 4). Numbers of awakenings were positively correlated between weeks, and total durations of awakenings were positively correlated between weeks. Within each week, number and total duration of awakenings were also positively correlated.

Between different weeks, however, there was an unexpected finding. Longer average total durations of awakenings per night at week 2 were significantly related to increases in number of lapses and median reaction time during week 12. Furthermore, the relation between average daily number of awakenings during week 2 and PVT lapses at week 12 was marginally significant $(\mathrm{r}=.280, p=.073)$. This may indicate that average nightly awakening duration during week 2 is a predictor of later neurocognitive deficits. A potential explanation for this finding is 
that the effect of sleep disruption on cognition is dose-dependent, with increased disruption causing increased deficits of longer duration (Banks, Van Dongen, Maislin \& Dinges, 2010).

Further analyses found that this relation between duration of awakenings at week 2 and PVT performance at week 12 was present for exclusively formula feeding participants (MRT, $\mathrm{r}=.866, p=.005$; lapses, $\mathrm{r}=.865, p=.006$ ), but not for exclusively breastfeeding participants (MRT, $\mathrm{r}=.093, p=.786$; lapses, $\mathrm{r}=.136, p=.691)$. This may support the finding that prolactin release during breastfeeding could function as a compensatory mechanism to protect against the negative neurocognitive effects of sleep disturbance. Prolactin, a protein necessary for breast milk production, peaks in concentration during the night (Ross, 2005) and levels further increase during and after breastfeeding (Kent, 2007). In non-human animals, higher prolactin levels in the bloodstream increase REM sleep (Stieger, 2003), which is associated with improved neurocognitive performance.

\section{Feeding Methods}

Research questions four and five investigated potential differences in number of awakenings or neurocognitive measures between women who exclusively breastfed and women who exclusively formula-fed.

Previous work on feeding methods and postpartum maternal sleep has found that exclusively breastfeeding mothers have more nocturnal awakenings at week 12 (Blyton, Sullivan, Edwards, 2007) and have a higher percentage of wake time after sleep onset at week 12 (Gay, Lee \& Lee, 2004). However, as expected from previous work on this dataset (Montgomery-Downs, Clawges, \& Santy, 2010), no differences were found in average objective number of awakenings per night based on feeding method. Differences between weeks 2 and 12 
were significant and very similar to those of research question two which included all postpartum women regardless of feeding method.

At week 12 participants had significantly more lapses and increased median reaction time on the PVT. There was no difference based on feeding method on number of lapses, however there was a significant difference based on feeding method for median reaction time. Exclusively formula feeding mothers $(414.5 \mathrm{~ms})$ had $70.6 \mathrm{~ms}$ slower reaction times overall compared to exclusively breastfeeding mothers (343.9 ms). By week 12, median reaction time for formula feeding mothers $(430.3 \mathrm{~ms})$ and breastfeeding mothers $(351.5 \mathrm{~ms})$ were still much higher than a normative reaction time of approximately 250ms for women (Blatter, Graw, Munch, Knoblauch, Wirz-Justice, \& Cajochen, 2006), indicating pronounced slowing of reaction times. This finding supports the prolactin hypothesis in that exclusively breastfeeding mothers had improved neurocognitive performance compared to exclusively formula feeding mothers at week 12 despite no differences in sleep characteristics. However, this performance difference was not expected to be present or detectable at week 2, as the protective effect of prolactin for breastfeeding women was proposed to be cumulative.

\section{Limitations}

Some limitations may have affected the findings of this study. For example, though activity data were gathered via actigraphy, which has been extensively validated as a measure of sleep/wake patterns (Benson, Friedman, Noda, Wicks, Wakabayashi, \& Yesavage, 2004; King, Jaffre, Morrish, Shneerson \& Smith, 2005; Sadeh, Sharkey \& Carskadon, 1994), the algorithm used to categorize awakenings has not been validated versus PSG, the gold standard measure of sleep/wake. Also, actigraphy generally underestimates wake because it cannot differentiate between immobile wakefulness and sleep, with an estimated $50 \%$ of normal awakenings spent 
immobile (Paquet, Kawinska, \& Carrier, 2007). Therefore, the numbers and durations of awakenings may have been underestimated and thresholds may have been overestimated in this study.

The control group used for this study also only completed one week of study procedures, while the postpartum women completed twelve weeks. While the PVT does show some minor improvements in performance with practice (Lim \& Dinges, 2008), overall postpartum women performed significantly worse over the course of the study.

Also, there were significant differences between postpartum women and controls on education level, income and percent living together. Though no direct connection between education level and reaction has been found, education level has been shown to be positively associated with general intelligence (Sewell and Shah, 1967), and general intelligence has been found to be associated with faster reaction time (Rindermann and Neubauer, 2004). This may indicate that the significantly higher education level of controls may have confounded our data by accounting for their faster reaction time compared to postpartum women. Income differences can be partially accounted for by a high percentage of the postpartum women in the living together category (and therefore having a combined household income), but income still may represent a potential confound.

Finally, by only using postpartum women who exclusively breastfed or formula fed, over half of all participants (57\%) could not be included in the analyses of research questions 4 and 5 . This resulted in a low $\mathrm{n}$ and low power for these research questions.

\section{Future Directions}

Future studies involving thresholds should incorporate male participants, especially postpartum fathers. Our control group consisted of females only, which is appropriate for 
comparison to postpartum women; however, the nocturnal awakening duration threshold for recall that was determined in controls may not be comparable to males.

Future studies should also attempt to include a more thorough battery of neurocognitive measures such as those used in a recent study of pregnant and postpartum cognition: the Concept Shifting Task, the Stroop Color-Word Interference Test, the Letter-Digit Substitution Test, and the Visual Verbal Word Learning Task (DeGroot, Vuurman, Hornstra and Jolles, 2006).

Also, further longitudinal analyses are needed on this dataset to investigate the relation between early increased duration of awakenings and later measures of neurocognitive performance.

\section{Conclusion}

Early parenthood is associated with neurocognitive performance deficits and sleep disturbance in the form of repeated awakenings for infant care and feeding. This study found worse reaction time for mothers who formula feed versus those who breastfeed, despite similar numbers of nocturnal awakenings. Also, early sleep disturbance in formula feeders was associated with later deficits in neurocognitive performance. These findings indicate that improved maternal cognition is positively related to breastfeeding, and that mothers who choose to formula feed should be especially protective of their sleep early in the postpartum period. This study also investigated memory for natural awakenings and found that slightly shorter durations of awakenings are necessary for recall than the current five minute rule of thumb, which may have implications for our understanding of insomnia and sleep state misperception. 


\section{References}

American Academy of Pediatrics, Policy Statement (2005) Breastfeeding and the use of human milk. Pediatrics, 115(2), 496-506.

Banks, S., Van Dongen, H. P. A., Maislin, G. \& Dinges, D. F. (2010) Neurobehavioral Dynamics Following Chronic Sleep Restriction: Dose-Response Effects of One Night for Recovery. Sleep, 33(8), 1013-1026.

Banks, S. \& Dinges, D. F. (2007) Behavioral and physiological consequences of sleep restriction. Journal of Clinical Sleep Medicine, 3(5), 519-528.

Benson K., Friedman, L., Noda, A., Wicks, D., Wakabayashi, E., \& Yesavage, J. (2004) The measurement of sleep by actigraphy: direct comparison of 2 commercially available actigraphs in a nonclinical population. Sleep, 27(5), 986-989.

Blatter, K., Graw, P., Munch, M., Knoblauch, V., Wirz-Justice, A. \& Cajochen, C. (2006) Gender and age differences in psychomotor vigilance performance under differential sleep pressure conditions. Behavioural Brain Research, 168, 312-317.

Blyton, D. M., Sullivan, C. E. \& Edwards, N. (2002) Lactation is associated with an increase in slow-wave sleep in women. Journal of Sleep Research, 11, 297-303.

Bonnet, M. H. (1983) Memory for events occurring during arousal from sleep. Psychophysiology, 20(1), 81-87.

Bonnet, M.H. (2005) Acute Sleep Deprivation. In M. H. Kryger, T. Roth, \& W. C. Dement (Eds), Principles and Practice of Sleep Medicine (Fourth Edition, pp. 51-66). Philadelphia, PA: Elsevier Saunders

Buckwalter, J. G., Buckwalter, D. K., Bluestein, B. W., \& Stanczyk, F. Z. (2001) Pregnancy and post partum: changes in cognition and mood. Progress in Brain Research, 133, 303-319. 
Carskadon, M.A., \& Dement, W.C. (2011) Chapter 2: Normal Human Sleep: An Overview. In M. H. Kryger, T. Roth, \& W. C. Dement (Eds), Principles and Practice of Sleep Medicine (Fifth Edition, pp. 16-26). Philadelphia, PA: Elsevier Saunders

Cartwright, R. D. (2004) The role of sleep in changing our minds: A psychologist's discussion of papers on memory reactivation and consolidation in sleep. Learning \& Memory, 11(6), $660-663$.

Connor, J., Whitlock, G., Norton, R., \& Jackson, R. (2001) The role of driver sleepiness in car crashes: a systematic review of epidemiological studies. Accident Analysis and Prevention, 33(1), 31-41.

Gay, C. L., Lee, K. A., \& Lee, S. Y. (2004) Sleep Patterns and Fatigue in New Mothers and Fathers. Biological Research for Nursing, 5(4), 311-318.

DeGroot, R. H. M, Vuurman, E. F. P. M, Hornstra, G. \& Jolles, J. (2006) Differences in Cognitive Performance during Pregnancy and Early Motherhood. Psychological Medicine, 36, 1023-1032.

Downey, R., \& Bonnet, M. H. (1987) Performance during frequent sleep disruption. Sleep, 10(4), 354-363.

Edinger, J. D. \& Krystal, A. D. (2003) Subtyping primary insomnia: is sleep state misperception a distinct clinical entity. Sleep Medicine Reviews, 7(3), 203-214.

Fifer, W. P., Byrd, D. L., Kaku, M., Eigsti, I. M., Isler, J. R., Grose-Fifer, J., Tarullo, A. R., \& Balsam, P. D. (2010) Newborn infants learn during sleep. Proceedings of the National Academy of Sciences, 107(22), 10320-10323.

Goodenough, D. R., Sapan, J., Cohen, H., Portnoff, G., \& Shapiro, A. (1971) Some experiments on the effects of sleep on memory. Psychophysiology, 8(6), 749-762. 
Henderson, J. M. T., France, K. G., Owens, J. L., \& Blampied, N. M. (2010) Sleeping through the night: the consolidation of self-regulated sleep across the first year of life. Pediatrics, 126(5), 1081-1087.

Ji, D., \& Wilson, M. A. (2007) Coordinated memory replay in the visual cortex and hippocampus during sleep. Nature Neuroscience, 10(1), 100-107.

Kent, J. C. (2007) How breastfeeding works. Journal of Midwifery and Womens Health, 52(6), 564-570.

King, M. A., Jaffre, M. O., Morrish, E., Shneerson, J. M., \& Smith, I. E. (2005) The validation of a new actigraphy system for the measurement of periodic leg movements in sleep. Sleep Medicine, 6(4), 1-7.

Landrigan, C. P., Rothschild, J. M., Cronin, J. W., Kaushal, R., Burdick, E., Katz, J. T., Lilly, C. M., Stone, P. H., Lockley, S. W., Bates, D. W., \& Czeisler, C. A. (2004) Effect of reducing interns' work hours on serious medical errors in intensive care units. New England Journal of Medicine, 351(18), 1838-1848.

Leger, D (1994) The cost of sleep-related accidents: a report for the National Commission on Sleep Disorders Research. Sleep, 17(1), 84-93.

Lim, J., Dinges, D. F. (2008) Sleep deprivation and vigilant attention. Annals of the New York Academy of Sciences, 1129, 305-322.

Lockley, S. W., Cronin, J. W., Evans, E. E., Cade, B. E., Lee, C. J., Landrigan, C. P., Rothschild, J. M., Katz, J. T., Lilly, C. M., Stone, P. H., Aeschbach, D., \& Czeisler, C. A. (2004) Effect of reducing intern's weekly work hours on sleep and attentional failures. New England Journal of Medicine, 351, 1829-1837. 
Montgomery-Downs, H. E., Clawges, H. M., \& Santy, E. E. (2010) Infant feeding methods and maternal sleep and daytime functioning. Pediatrics, 126(6), 1562-1568.

Montgomery-Downs, H. E., Insana, S. P., Clegg-Kraynok, M. M., \& Mancini, L. M. (2010) Normative longitudinal maternal sleep: the first 4 postpartum months. American Journal of Obstetrics and Gynecology, 203(5), 465.e1-465.e7.

Orzel-Gryglewska, J. (2010) Consequences of sleep deprivation. International Journal of Occupational Medicine and Environmental Health, 23(1), 95-114.

Paquet, J., Kawinska, A. \& Carrier, J. (2007) Wake detection capacity of actigraphy during sleep. Sleep, 30(10), 1362-1369.

Pennartz, C. M. A., Uylings, H. B. M., Barnes, C. A., \& McNaughton, B. L. (2002) Memory reactivation and consolidation during sleep: from cellular mechanisms to human performance. Progress in Brain Research, 138, 143-166.

Perlis, M. L., Smith, M. T., Orff, H. J., Andrews, P. J., \& Giles, D. E. (2001) The mesograde amnesia of sleep may be attenuated in subjects with primary insomnia. Physiology \& Behavior, 74, 71-76.

Portnoff, G., Baekeland, F., Goodenough, D. R., Karacan, I., \& Shapiro, A. (1966) Retention of verbal materials perceived immediately prior to onset of non-REM sleep. Perceptual and Motor Skills, 22, 751-758.

Radloff, L. S. (1977) The CES-D scale: A self-report depression scale for research in the general population. Applied Psychological Measure, 1, 385-401.

Rindermann, H. \& Neubauer, A. C. (2004) Processing speed, intelligence, creativity, and school performance: Testing of causal hypotheses using structural equation models. Intelligence, $32,573-589$. 
Rosekind, M. R. (2005) Underestimating the societal costs of impaired alertness: safety, health and productivity risks. Sleep Medicine, 6(1), S21-S25.

Roky, R., Obál, F., Valatx, J., Bredow, S., Fang, J., Pagano, L., \& Krueger, J. M. (1995) Prolactin and rapid eye movement sleep regulation. Sleep, 18(7), 536-542.

Ross, L. E., Murray, B. J., \& Steiner, M. (2005) Sleep and perinatal mood disorders: a critical review. Journal of Psychiatry and Neuroscience, 30(4), 247-256.

Sadeh, A., Sharkey, K., \& Carskadon, M. (1994) Activity-based sleep-wake identification: an empirical test of methodological issues. Sleep, 17, 201-207.

Sewell, W. H. \& Shah, V. P. (1967) Socioeconomic Status, Intelligence, and the Attainment of Higher Education. Sociology of Education, 40(1), 1-23.

Shapiro, A., Goodenough, D. R., \& Gryler, R. B. (1973) Dream recall as a function of method of awakening. Psychosomatic Medicine, 25(2), 174-180.

Shapiro, C. M., \& Dement, W. C. (1993) Impact and epidemiology of sleep disorders. British Medical Journal, 306, 1604-1607.

Stepanski, E. J. (2002) The effect of sleep fragmentation on daytime function. Sleep, 25(3), 268276.

Stieger, A. (2003) Sleep and endocrinology. Journal of Internal Medicine, 254, 13-22.

Takehara-Nishiuchi, K., \& McNaughton, B. L. (2010) Spontaneous changes of neocortical code for associative memory during consolidation. Science, 322, 960-963.

Thomas, K. A., \& Foreman, S. W. (2005) Infant sleep and feeding pattern: effects on maternal sleep. Journal of Midwifery \& Women's Health, 50, 399-404.

Wu, T., \& Chen, P. (2009) Health consequences of nutrition in childhood and early infancy. Pediatrics \& Neonatology, 50(4), 135-142. 
Wyatt, J. K., Bootzin, R. R., Anthony, J., \& Bazant, S. (1994) Sleep onset is associated with retrograde and anterograde amnesia. Sleep, 17(6), 502-511.

Wyatt, J. K., Bootzin, R. R., Allen, J. J. B., \& Anthony, J. L. (1997) Mesograde amnesia during the sleep onset transition: replication and electrophysiological correlates. Sleep, 20(7), $512-522$. 
Table 1

Summary of Studies Investigating Memory During the Sleep/Wake Transition

\begin{tabular}{l|c|c} 
Transition Period & Study & Finding \\
\hline Forced Awakening & Portnoff et al. (1966) & NREM interferes \\
Goodenough et al. (1971) & 5 minutes \\
Bonnet (1983) & Stage awakened from \\
Sleep Onset & Wyatt et al. (1994) & 3 minutes (recall) \\
& Wyatt et al. (1997) & 4 minute (recognition) \\
& & None (recognition) \\
\hline
\end{tabular}


Table 2

Demographics by Group

\begin{tabular}{|c|c|c|}
\hline Demographics & Postpartum & Control \\
\hline$N$ & 44 & 27 \\
\hline \multicolumn{3}{|l|}{ Age (years) } \\
\hline$M(S D)$ & $26.8(4.1)$ & $27.8(6.1)$ \\
\hline Range & $18.4-35.5$ & $20.5-44.2$ \\
\hline \multicolumn{3}{|l|}{ Education (years) } \\
\hline$M(S D)$ & $15.9(2.8)$ & $19.0(2.8)^{\star \star}$ \\
\hline Range & $10-22$ & $14-25$ \\
\hline \multicolumn{3}{|l|}{ Income } \\
\hline$M(S D)$ & $\$ 61,750(34,354)$ & $\$ 31,652(\$ 30,814)^{*}$ \\
\hline Range & $\$ 12,000-\$ 150,000$ & $\$ 5,000-\$ 110,000$ \\
\hline Married/Living Together & $86.4 \%$ & $22.2 \%$ ** \\
\hline Caucasian & $88.6 \%$ & $100.0 \%$ \\
\hline
\end{tabular}

${ }^{\star} p<.005,{ }^{\star \star} p<.001$ 
Table 3

Median Ratio of Subjective to Objective Number of Postpartum Awakenings by Week

\begin{tabular}{c|c|c|c}
\hline \multirow{2}{*}{} & \multicolumn{2}{|c|}{ Median Number of Awakenings (IQR) } & Median Ratio (IQR) \\
\cline { 2 - 4 } & Subjective & Objective & (Subj/Obj) \\
\hline Week 2 & $2.9(1.4)$ & $5.7(2.9)$ & $.52(.31)$ \\
Week 12 & $2.1(2.0)$ & $3.0(3.0)$ & $.79(.78)$ \\
\hline Controls & $2.2(2.8)$ & $1.5(2.8)$ & $1.2(1.2)$ \\
\hline
\end{tabular}


Table 4

Correlations between Number and Duration of Awakenings and Performance Variables at Weeks 2 and 12
1.
2.
3.
4.
5.
6.
7.
8.

Number of

Awakenings

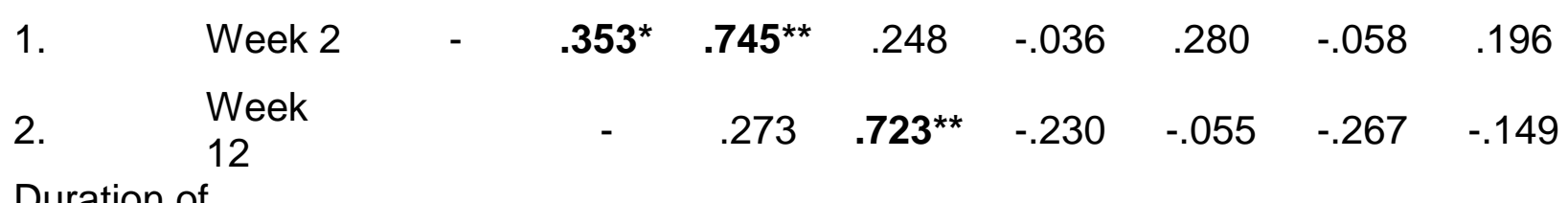

Duration of

Awakenings

3.

Week 2

$.429 * * \quad .128 \quad .430 * * \quad .166 \quad .353^{*}$

4.

Week

PVT

12

$\begin{array}{lllll}- & -.094 & .187 & -.161 & .090\end{array}$

Lapses

5.

Week 2

- $.691^{* *} .922^{* *} .698^{* *}$

6.

Week

12

$-.651^{* *} .939 * *$

PVT Median Reaction Time

7. Week 2

$-.735^{\star *}$

8.

Week

12

${ }^{\star} p<.05$ (two-tailed), ${ }^{\star \star} p<.01$ (two-

tailed) 


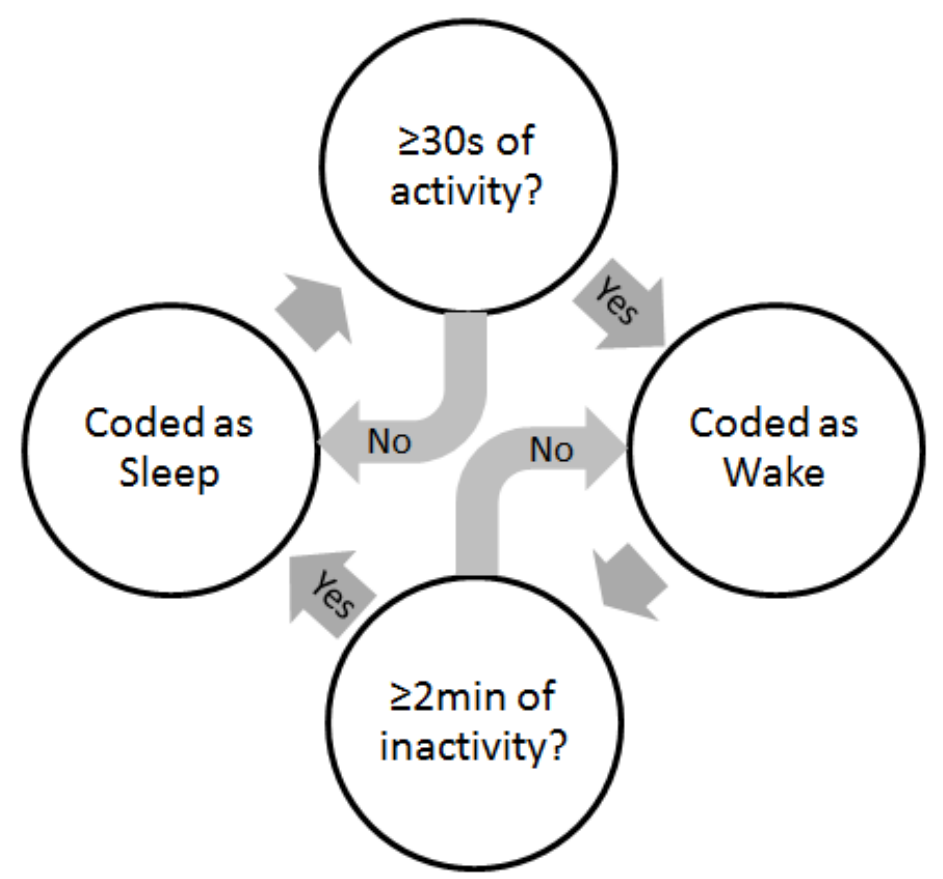

Figure 1. Flowchart of algorithm coding raw actigraphy data as Sleep or Wake based on 30 s of activity separated by 2 minutes of inactivity. 


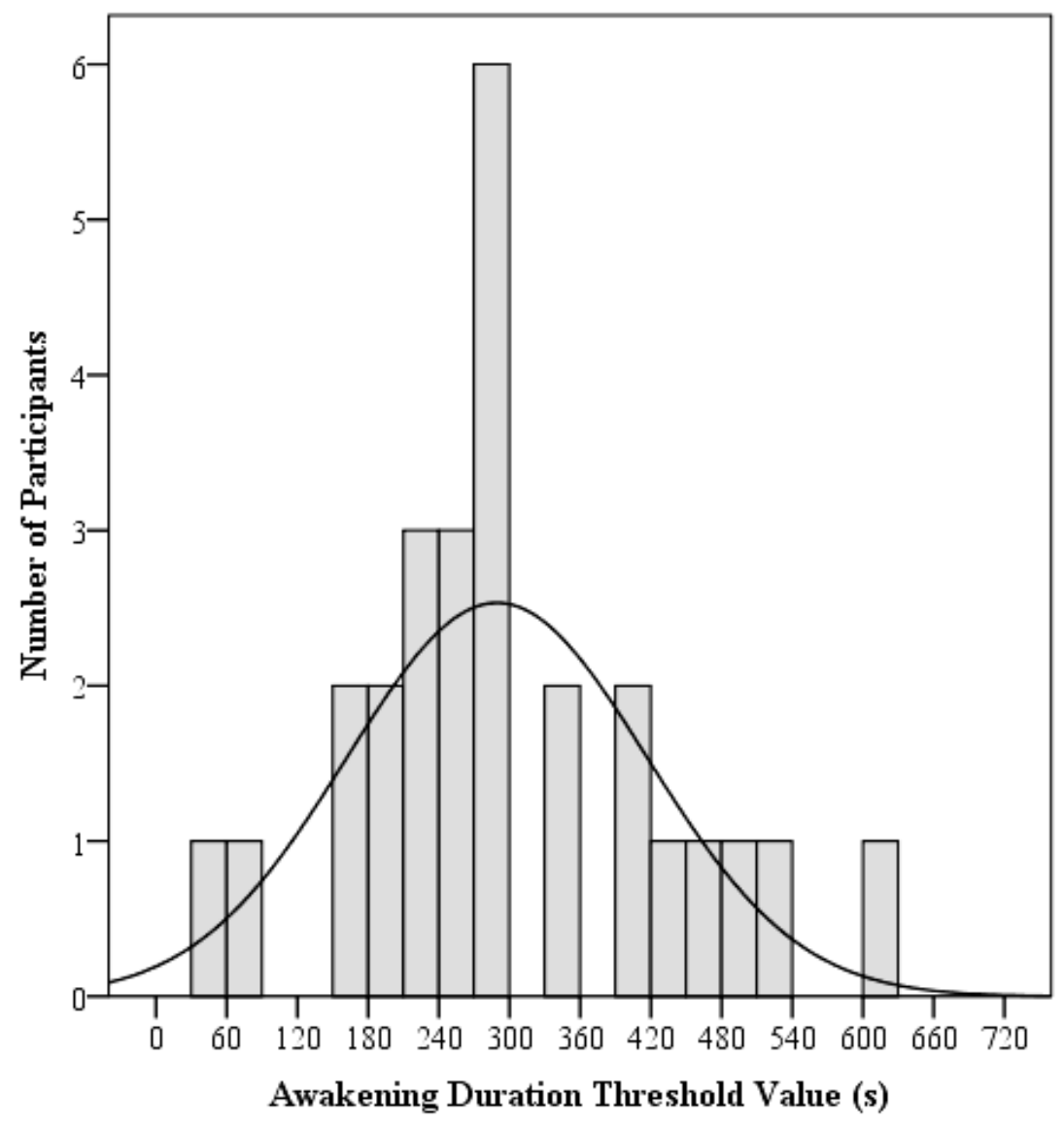

Figure 2. Frequency histogram of controls' awakening duration threshold values in seconds with a normal curve. 


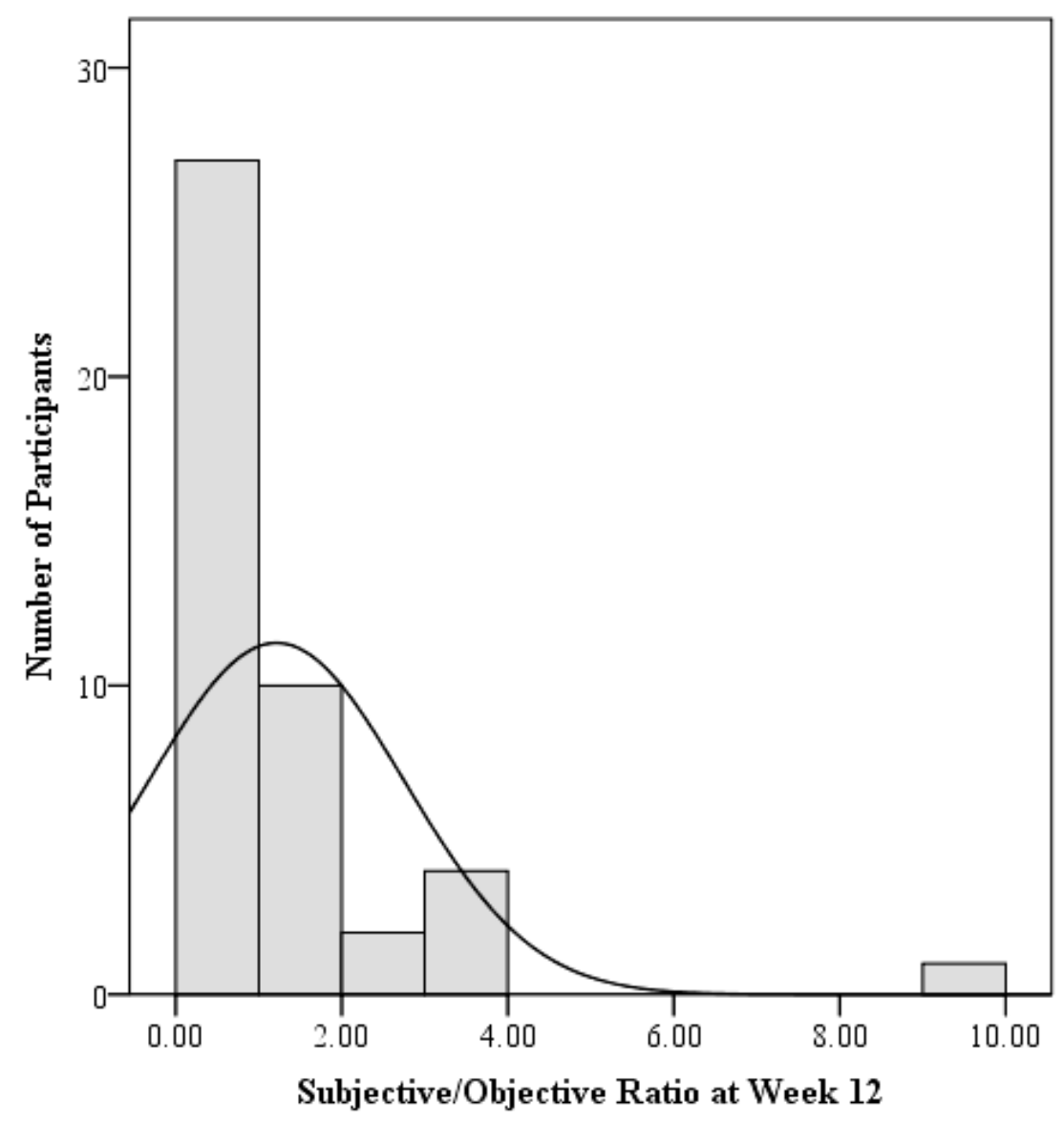

Figure 3. Histogram of the ratio of subjective to objective number of awakenings at week 12. 


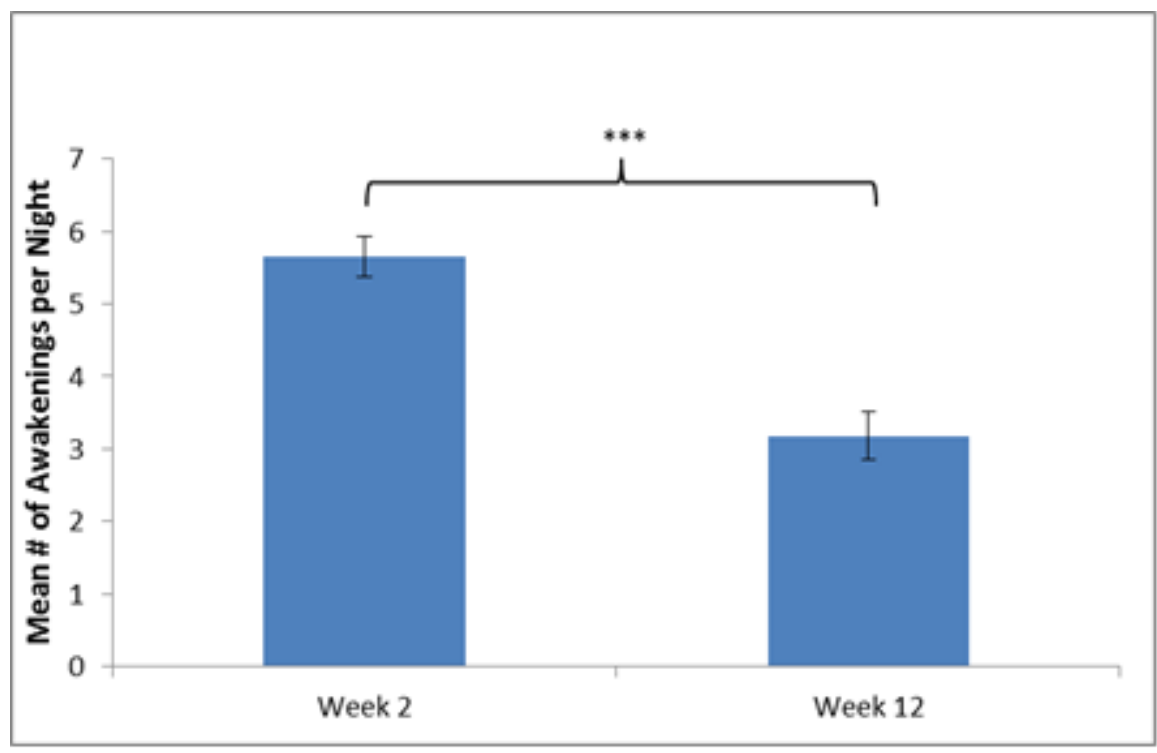

Figure 4. Mean objective number of awakenings per night with standard error bars measured during postpartum week 2 and 12 . $* * * p<.001$ 


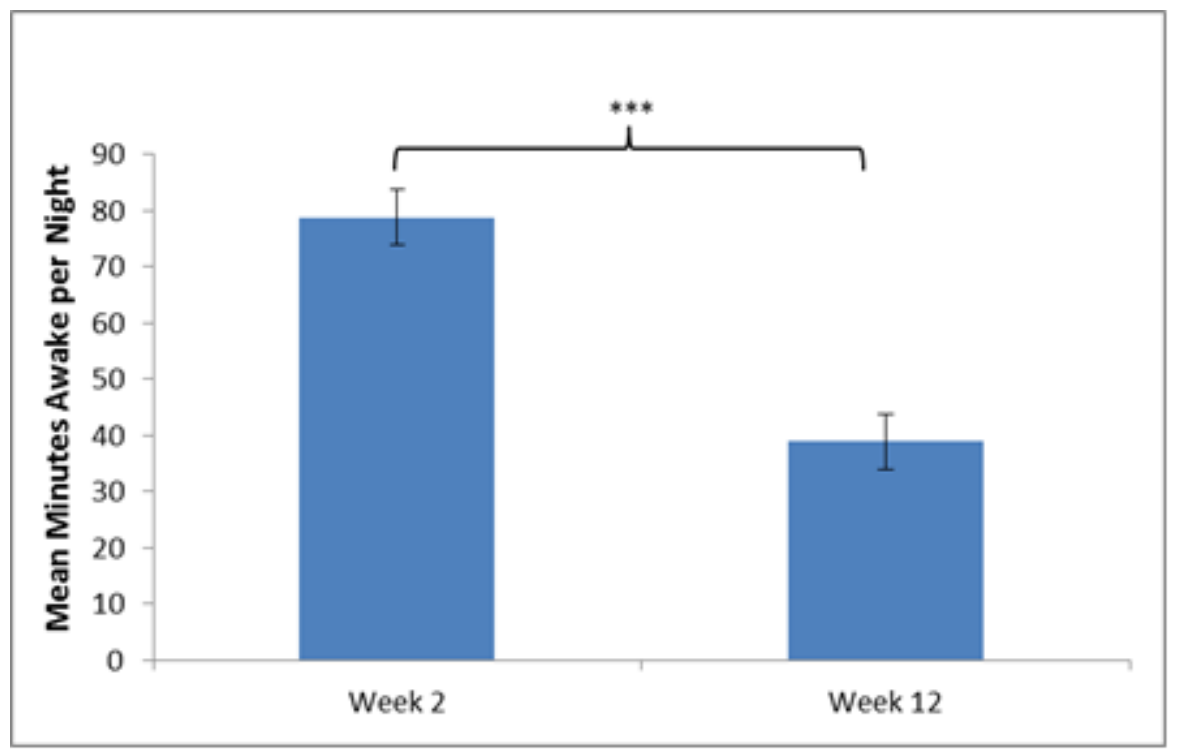

Figure 5. Mean objective duration of awakenings per night with standard error bars measured during postpartum week 2 and $12 . * * * p<.001$ 


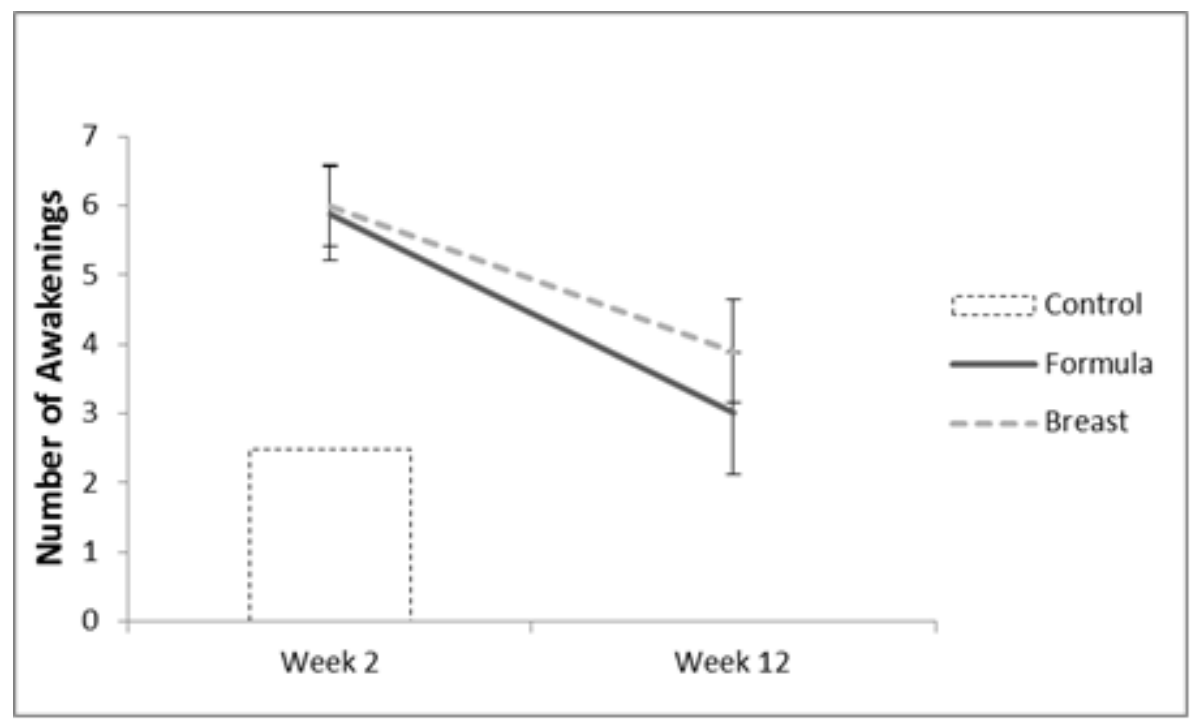

Figure 6. Objective number of awakenings per night at postpartum week 2 and 12 with separate lines by feeding method. For reference, a dashed bar indicates number of awakenings for the one week control group. 


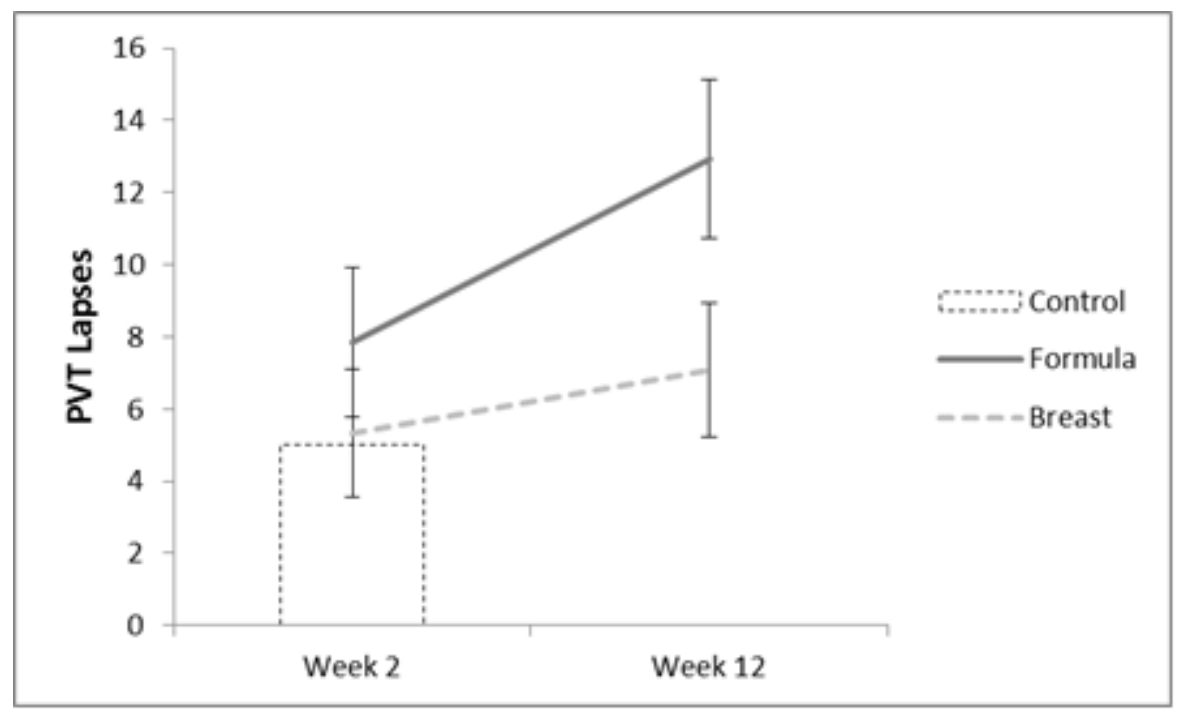

Figure 7. PVT lapses with error bars at postpartum weeks 2 and 12 with separate lines by feeding method. For reference, a dashed bar indicates PVT lapses for the one week control group. 


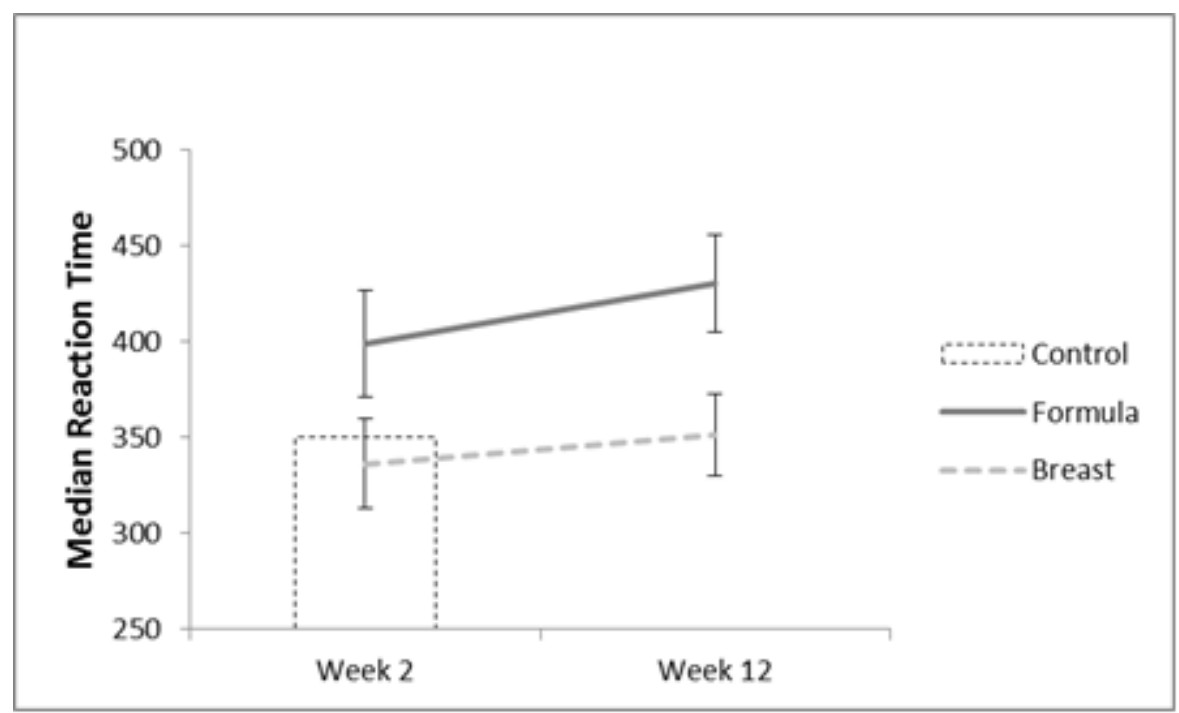

Figure 8. PVT median reaction time at postpartum weeks 2 and 12 with separate lines by feeding method. For reference, a dashed bar indicates median reaction time for the one week control group. 


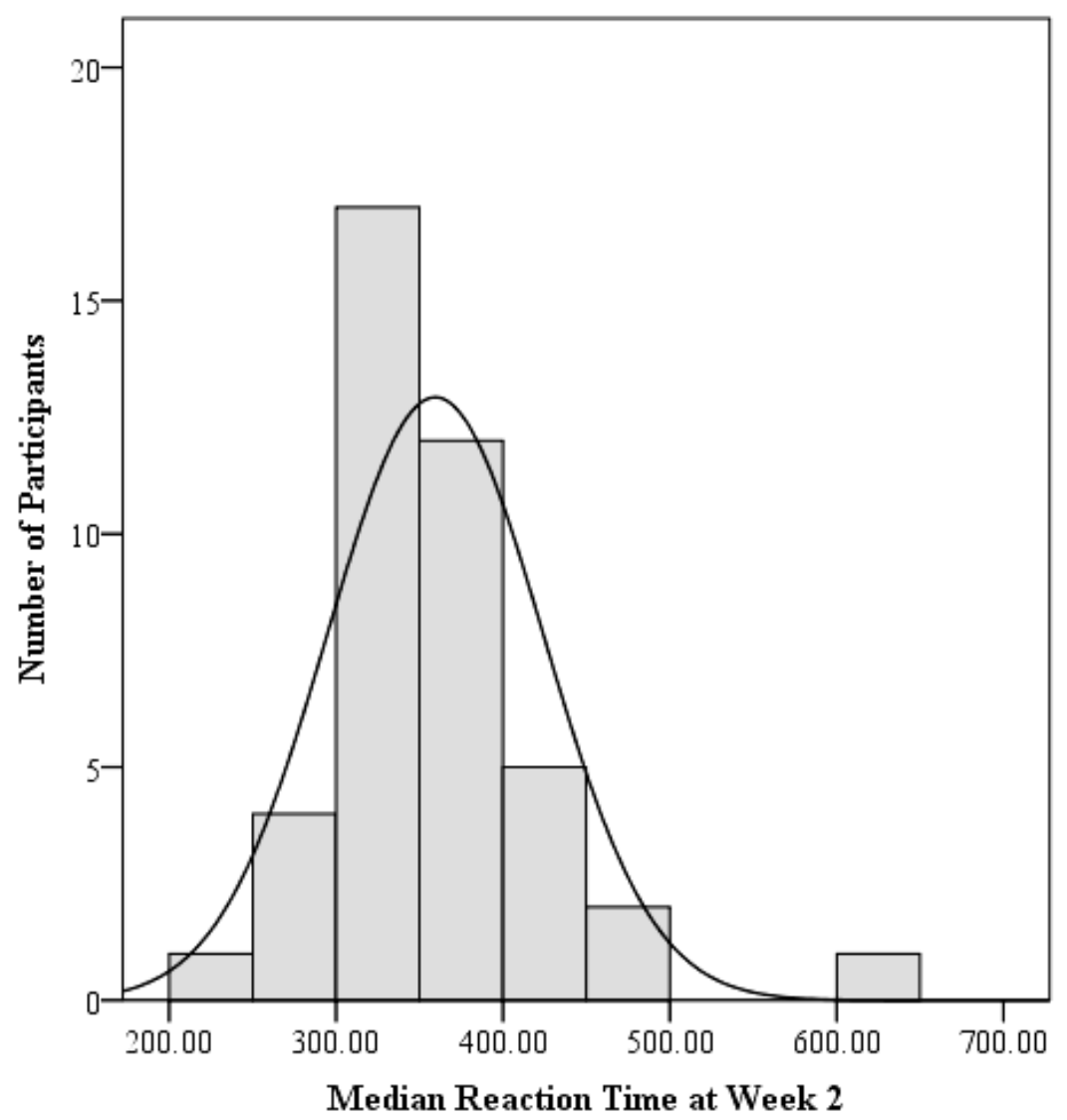

Figure 9. Histogram of median reaction time at week 2. 
Appendix A: Number of Awakenings PDA Prompt

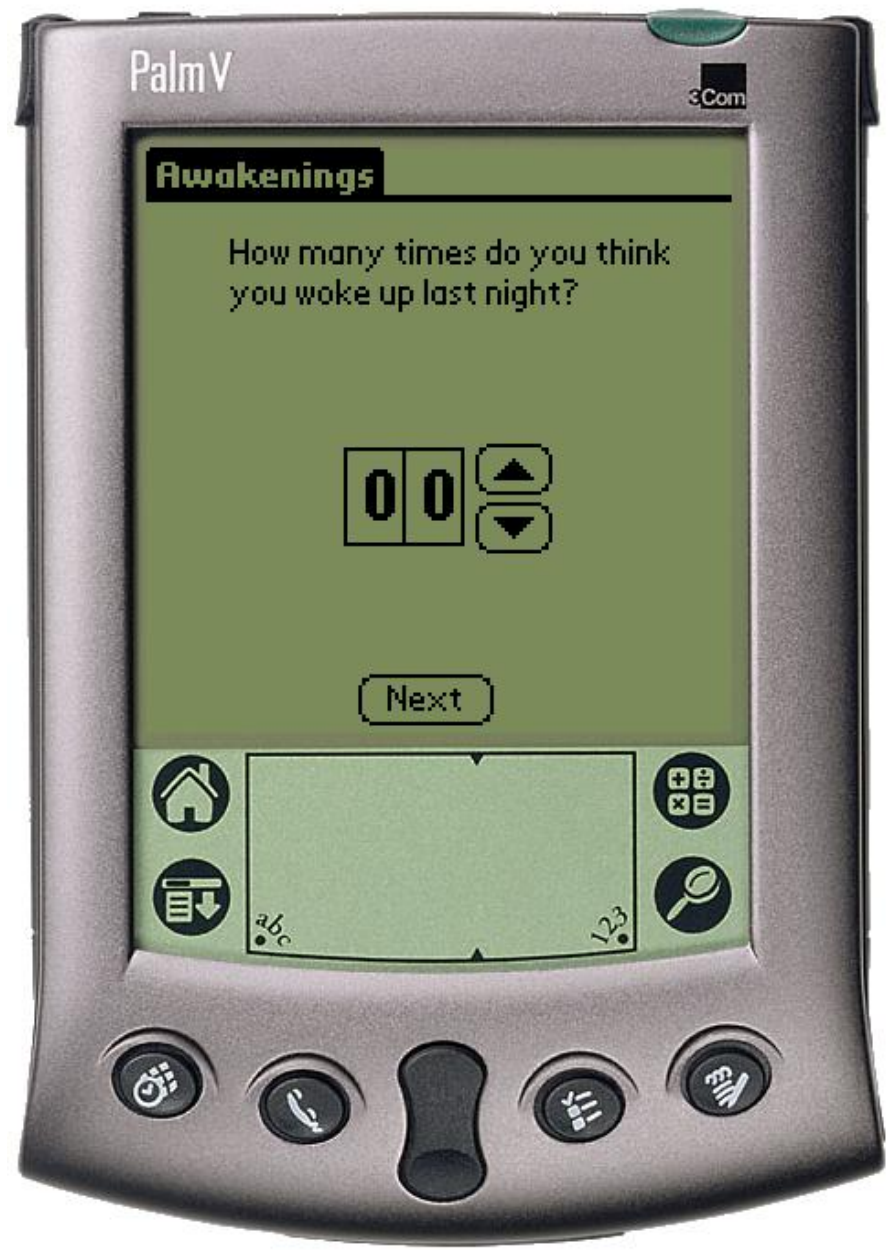


Appendix B: Duration of Awakenings PDA Prompt

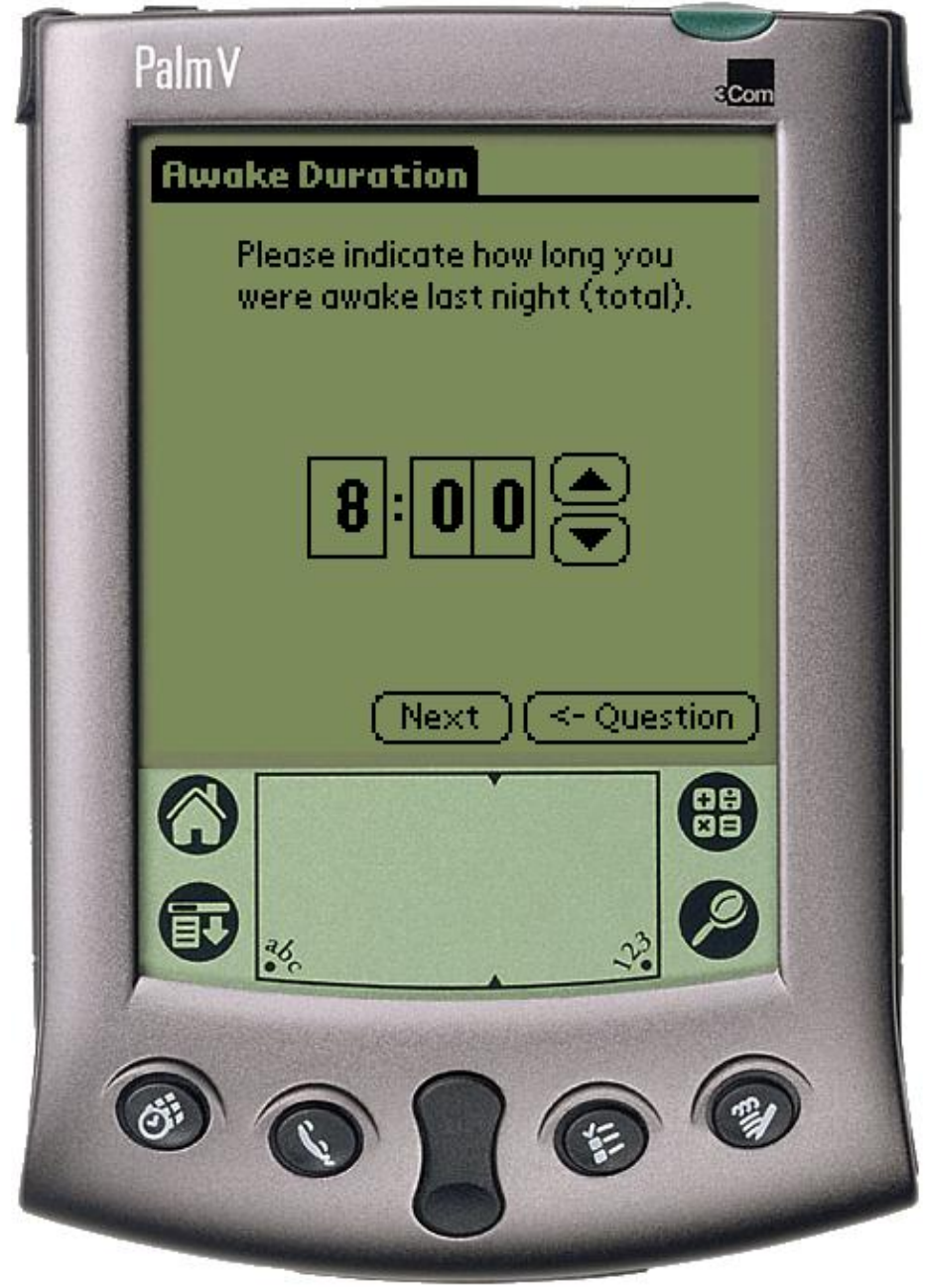


Appendix C: Psychomotor Vigilance Test

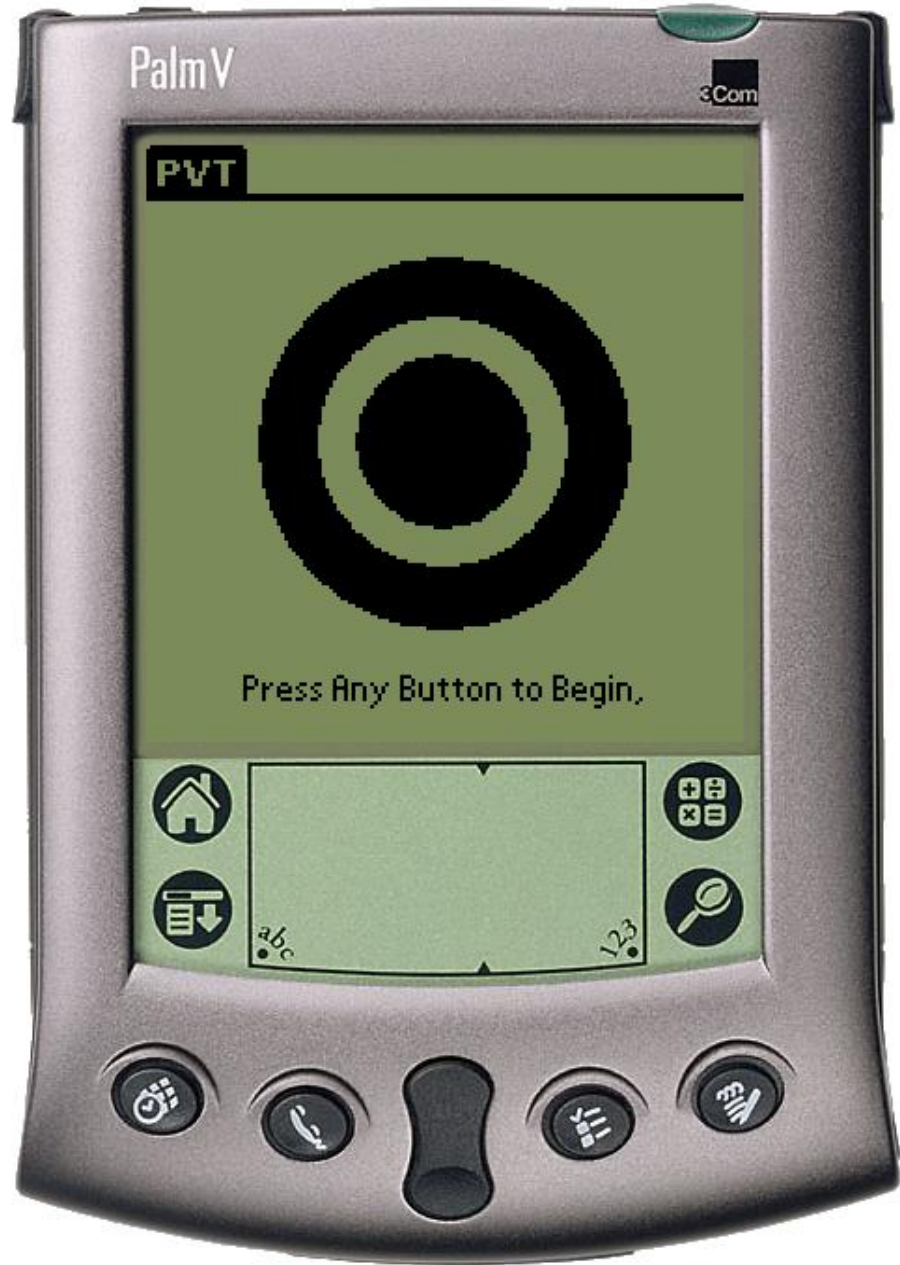




\section{Appendix D: Screening Dialogue and CES-D}

\section{MSD SCREEN}

\section{Date}

Hello, my name is __. Thank you for your interest in the WVU study on Matemal Sleep Disturbance. I'm happy to answer any questions you have about the study and then I need to ask you some questions to make sure that you're eligible for the study.

\section{[ANSWER QUESTIONS.]}

How did you hear about the study?

Are you currently pregnant? Yes No

Is this your first baby? Yes No

If YES to both - Congratulations! When is your due date?

If NO to either - I'm sorry, the study is for women who are expecting their first baby. I really appreciate your calling and if you know of anyone who is expecting I hope you will ask them to call us. [END]

It will take about 5 minutes for me to ask you some questions about how you have been feeling during the past week. Answering these questions is entirely voluntary. You may refuse to answer any question or stop at any time without penalty. If your answers indicate that you may benefit from community resources for depression I will tell you at the end and give you information about such resources. Your answers will not be used for research and will be destroyed after we're done talking.

Do you agree to answer some questions? Yes

No

IF NO: May I ask why not? [END]

\section{AFTER CES-D, IF QUALIFYING:}

Name:

Telephone:

Address:

City, State, ZIP:

Appointment: 


\section{Appendix D (continued)}

I'm going to ask you 20 questions about ways you may have felt or behaved recently. For each statement, please tell me which option describes how often you have felt this way during the past week. The choices are:

\section{Rarely or Not at All}

Some of the Time

Occasionally

Most of the Time

\section{SHRED THIS FORM AFTER PHONE CALL}

\begin{tabular}{|c|c|c|c|c|}
\hline DURING THE PAST WEEK: & $\begin{array}{l}\text { Rarely or } \\
\text { Not at All }\end{array}$ & $\begin{array}{l}\text { Some of } \\
\text { the Time }\end{array}$ & Occasionally & $\begin{array}{l}\text { Most of } \\
\text { the Time }\end{array}$ \\
\hline 1. I was bothered by things that don't usually bother me. & 0 & 1 & 2 & 3 \\
\hline 2. I did not feel like eating, my appetite was poor. & 0 & 1 & 2 & 3 \\
\hline $\begin{array}{l}\text { 3. I felt that I could not shake the blues even with the help } \\
\text { from my family and friends. }\end{array}$ & 0 & 1 & 2 & 3 \\
\hline 4. I felt that I was just as good as other people. & 3 & 2 & 1 & 0 \\
\hline 5. I had trouble keeping my mind on what I was doing. & 0 & 1 & 2 & 3 \\
\hline 6. I felt depressed. & 0 & 1 & 2 & 3 \\
\hline 7. I felt that everything I did was an effort. & 0 & 1 & 2 & 3 \\
\hline 8. I felt hopeful about the future. & 3 & 2 & 1 & 0 \\
\hline 9. I thought my life had been a failure. & 0 & 1 & 2 & 3 \\
\hline 10. I felt fearful. & 0 & 1 & 2 & 3 \\
\hline 11. My sleep was restless. & 0 & 1 & 2 & 3 \\
\hline 12. I was happy. & 3 & 2 & 1 & 0 \\
\hline 13. I talked less than usual. & 0 & 1 & 2 & 3 \\
\hline 14. I felt lonely. & 0 & 1 & 2 & 3 \\
\hline 15. People were unfriendly. & 0 & 1 & 2 & 3 \\
\hline 16. I enjoyed life. & 3 & 2 & 1 & 0 \\
\hline 17. I had crying spells. & 0 & 1 & 2 & 3 \\
\hline 18. I felt sad. & 0 & 1 & 2 & 3 \\
\hline 19. I felt that people dislike me. & 0 & 1 & 2 & 3 \\
\hline 20. I could not "get going". & 0 & 1 & 2 & 3 \\
\hline
\end{tabular}

SUBTOTALS:

SUM 


\section{Appendix D (continued)}

Finally, are you currently being treated for depression or anxiety? This includes over the counter medications like St. John's Wort. YES NO

Thank you very much for answering these questions.

[IF THE PERSON MEETS CRITERIA FOR MSD, SAY]: It sounds as though you might meet criteria for the Maternal Sleep Disturbance Study. Dr. Montgomery-Downs will be calling you in the next few days to follow up. Is there a good day or time for her to reach you?

[IF THE PERSON DOESN'T MEET CRITERIA FOR MSD, AND HAS CES-D >16, SAY]: From your answers it sounds as though you don't meet criteria for the MSD Study because you've scored above our threshold for symptoms of depression. I'd like to give you some information about community resources that might help you? Would you like me to give you these over the phone or to send them to you?

If PHONE:

If SEND:

Address:

[ASK REGARDLESS OF WHETHER PERSON MEETS CRITERIA]: May we contact you about other opportunities to participate in research? Answering yes does not mean that you agree to participate in future studies.

If YES:

Name:

Phone: 
Appendix E: Referral List

\section{REFERRALS}

\section{Elizabeth Cohen, LICSW}

West Virginia University Medical Corp.

POBox 782

Morgantown, WV 26507-0782

(304) 598-6868

cohene@rrcbhsc.wvu.edu

Chestnut Ridge Hospital

930 Chestnut Ridge Road

Morgantown, WV 26505

(304) 598-6400

Fremouw Psychological Associates

1137 Van Voorhis Road

Morgantown, WV

(304) $598-2300$

\section{The Quin Curtis Center}

Department of Psychology

West Virginia University

Life Sciences Building, Rooms 1331-1341

53 Campus Drive

Morgantown, WV 26506-6040

(304) 293-2001, ext 31671

Valley Health Care

301 Scott Avenue

Morgantown, WV

(304) $296-1731$

Wedgewood Family Practice \& Psychiatry Associates, Inc.

1197 Van Voorhis Rd.

Morgantown, WV

(304) 599-1975

Ruby MemorialHospital - Emergency Department

West Virginia University Health Sciences Center, 1 Medical Center Dr.

Morgantown, WV

(304) 598-4171

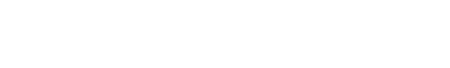

